Review

\title{
Conductive Atomic Force Microscopy of Semiconducting Transition Metal Dichalcogenides and Heterostructures
}

\author{
Filippo Giannazzo *D, Emanuela Schilirò, Giuseppe Greco $\mathbb{D}^{\mathbb{D}}$ and Fabrizio Roccaforte $\mathbb{D}$ \\ CNR-IMM, Strada VIII, 5-5121 Catania, Italy; emanuela.schiliro@imm.cnr.it (E.S.); \\ giuseppe.greco@imm.cnr.it (G.G.); fabrizio.roccaforte@imm.cnr.it (F.R.) \\ * Correspondence: filippo.giannazzo@imm.cnr.it
}

Received: 31 March 2020; Accepted: 17 April 2020; Published: 22 April 2020

check for updates

\begin{abstract}
Semiconducting transition metal dichalcogenides (TMDs) are promising materials for future electronic and optoelectronic applications. However, their electronic properties are strongly affected by peculiar nanoscale defects/inhomogeneities (point or complex defects, thickness fluctuations, grain boundaries, etc.), which are intrinsic of these materials or introduced during device fabrication processes. This paper reviews recent applications of conductive atomic force microscopy (C-AFM) to the investigation of nanoscale transport properties in TMDs, discussing the implications of the local phenomena in the overall behavior of TMD-based devices. Nanoscale resolution current spectroscopy and mapping by C-AFM provided information on the Schottky barrier uniformity and shed light on the mechanisms responsible for the Fermi level pinning commonly observed at metal/TMD interfaces. Methods for nanoscale tailoring of the Schottky barrier in $\mathrm{MoS}_{2}$ for the realization of ambipolar transistors are also illustrated. Experiments on local conductivity mapping in monolayer $\mathrm{MoS}_{2}$ grown by chemical vapor deposition (CVD) on $\mathrm{SiO}_{2}$ substrates are discussed, providing a direct evidence of the resistance associated to the grain boundaries (GBs) between $\mathrm{MoS}_{2}$ domains. Finally, C-AFM provided an insight into the current transport phenomena in TMD-based heterostructures, including lateral heterojunctions observed within $\mathrm{Mo}_{\mathrm{x}} \mathrm{W}_{1-\mathrm{x}} \mathrm{Se}_{2}$ alloys, and vertical heterostructures made by van der Waals stacking of different TMDs (e.g., $\mathrm{MoS}_{2} / \mathrm{WSe}_{2}$ ) or by CVD growth of TMDs on bulk semiconductors.
\end{abstract}

Keywords: conductive atomic force microscopy; transition metal dichalcogenides; Schottky barrier; conductivity; heterostructures

\section{Introduction}

In the last years, transition metal dichalcogenides (TMDs) have attracted an increasing scientific interest because of their unique and tunable electronic structure, holding great promise for next generation applications in electronics and optoelectronics [1,2]. These layered materials are composed by the stacking of $M X_{2}$ trilayers $(M=M o, W$, etc. and $X=S, S e, T e$, etc.) held together by van der Waals bonds. Each trilayer consists of a layer of hexagonal-packed transition-metal atoms (M) sandwiched between two layers of chalcogen atoms $(\mathrm{X})$, with a strong intralayer covalent bond between the different atoms. Depending on the composition and internal structure of the individual trilayers, the electronic behavior of TMDs can range from insulating to semiconducting and semi-metallic. Furthermore, the optical and electronic properties of these layered materials depend on the stacking order and number of layers [3]. For instance, bulk or multilayer $\mathrm{MoS}_{2}$ exhibit an indirect band gap of $\sim 1.3 \mathrm{eV}$, whereas monolayer $\mathrm{MoS}_{2}$ presents a direct band gap of 1.8-1.9 eV. A similar behavior also occurs in other semiconducting TMDs. The tunable band gap along with the high quantum efficiency and optical 
absorption make TMDs interesting for optoelectronics [1]. In addition, due to the superior electrostatic control of channel conductance in field effect transistors (FETs) with an ultra-thin TMD body [4,5], these $2 \mathrm{D}$ semiconductors are currently regarded as strategic materials for the post-Si complementary metal oxide semiconductor (CMOS) technology [6]. Very interesting performances in terms of the on/off current ratio $\left(10^{6}-10^{8}\right)$ and a subthreshold swing approaching the ideal value (60 meV/decade) have been already demonstrated in the early studies on single layer [4] and multilayer MoS 2 FETs [5]. More recently, ultra-scaled $\mathrm{MoS}_{2}$ FETs with a $1 \mathrm{~nm}$ gate length [7] or with a sub -10 nm channel length [8] have been reported, further supporting the expectations for $\mathrm{MoS}_{2}$ transistors to further extend the validity of Moore's law [9]. In addition, as replacements of silicon for the CMOS technology, TMDs are also currently employed as building blocks of new transistor concepts based on vertical van der Waals heterostructures [10-12], or in-plane heterojunctions obtained by engineering the TMDs composition [13] or even the dielectric environment of the 2D semiconductors [14].

To date, many of these TMD-based (opto)electronic devices have been fabricated using small flakes obtained by mechanical exfoliation from the parent bulk crystals, due to the ease of preparation in academic laboratories [4,10]. However, the lateral size (few micrometres) and the low density of flakes produced by this approach are unsuitable for practical applications, and large area deposition methods of TMDs are required for a scale-up of these technologies. In the last years, many progresses have been made in the growth of TMDs by chemical vapor deposition (CVD). The direct CVD growth of TMDs, in particular $\mathrm{MoS}_{2}$, on noncatalytic insulating or semiconducting substrates (including $\mathrm{SiO}_{2}$ [15-17], sapphire [18], and GaN [19]) is possible at relatively low temperatures in the range from 700 to $900{ }^{\circ} \mathrm{C}$. This represents an important aspect for the integration of this process in the CMOS devices fabrication flow. The CVD growth of highly uniform monolayer TMDs on $100 \mathrm{~mm}$ [20] and, more recently, $150 \mathrm{~mm}$ wafers [21] have been demonstrated. Furthermore, alternative chemical deposition methods, e.g., atomic layer deposition (ALD) [22], or physical depositions, e.g., molecular beam epitaxy (MBE) [23] and pulsed laser deposition (PLD) [24], are currently explored to achieve layer-by-layer growth of $\mathrm{MoS}_{2}$ on a large area, up to the wafer scale.

Table 1 summarizes some of the most relevant achievements in the TMD devices and materials growth in the last years.

Table 1. Recent progresses in the transition metal dichalcogenide (TMD) materials growth and devices.

\begin{tabular}{|c|c|c|}
\hline Year & Achievements & Ref. \\
\hline 2011 & Monolayer $\mathrm{MoS}_{2}$ n-type FET with a high-k top gate dielectric & [4] \\
\hline 2011 & Integrated logic circuit based on a single-layer $\mathrm{MoS}_{2}$ & [25] \\
\hline 2012 & Monolayer WSe $_{2}$ p-type FET with a high-k top gate dielectric & [26] \\
\hline 2012 & Chemical vapor deposition (CVD) of $\mathrm{MoS}_{2}$ atomic layers on $\mathrm{SiO}_{2}$ substrates & [15] \\
\hline 2014 & FET built from all 2D material components & [27] \\
\hline 2014 & Vertical and in-plane heterostructures of $\mathrm{WS}_{2} / \mathrm{MoS}_{2}$ monolayers grown by CVD & [13] \\
\hline 2014 & Atomic layer deposition (ALD) of $\mathrm{MoS}_{2}$ thin films & [22] \\
\hline 2015 & CVD of epitaxial monolayer $\mathrm{MoS}_{2}$ on sapphire & [18] \\
\hline 2015 & Metal organic CVD of a highly uniform monolayer $\mathrm{MoS}_{2}$ on $100 \mathrm{~mm} \mathrm{SiO}{ }_{2}$ wafers & [20] \\
\hline 2015 & $\mathrm{WSe}_{2}$-based CMOS and integrated circuits & [28] \\
\hline 2015 & Tunnel diodes based on $\mathrm{MoS}_{2} / \mathrm{WSe}_{2}$ vdW heterostructures grown by CVD & [12] \\
\hline 2016 & $\mathrm{MoS}_{2}$ FET with a $1 \mathrm{~nm}$ gate length & [7] \\
\hline 2016 & $\mathrm{MoS}_{2}$ FET with a sub $-10 \mathrm{~nm}$ channel length & [8] \\
\hline 2016 & CVD of epitaxial monolayer $\mathrm{MoS}_{2}$ on gallium nitride & [18] \\
\hline 2016 & Pulsed laser deposition (PLD) of $\mathrm{MoS}_{2}$ thin films & [24] \\
\hline 2017 & Ambipolar $\mathrm{MoS}_{2}$ FET by tailoring the Schottky barrier with oxygen plasma & [29] \\
\hline 2017 & Ohmic contacts to monolayer $\mathrm{MoS}_{2}$ by van der Waals bonded metal/h-BN electrodes & [30] \\
\hline 2017 & Demonstration of a microprocessor based on CVD grown bilayer $\mathrm{MoS}_{2}$ & [31] \\
\hline 2017 & Hot electron transistor with GaN emitter, graphene base, and $\mathrm{WSe}_{2}$ base-collector barrier & [32] \\
\hline 2018 & Batch CVD growth of uniform monolayer $\mathrm{MoS}_{2}$ on $150 \mathrm{~mm}$ soda-lime glass wafers & [21] \\
\hline 2019 & Van der Waals contacts between 3D metals and 2D semiconducting TMDs & [33] \\
\hline
\end{tabular}


In spite of these recent progresses, several issues need to be addressed to fully exploit the potential of TMDs in real applications. In addition to the request of optimized thickness uniformity, defectivity, and doping of the deposited films, a further challenge for materials growth is represented by the engineering of TMD vertical or lateral heterojunctions [12]. On the other hand, low resistance ohmic contacts and selective-area doping of TMDs are two major requirements for the fabrication of devices based on these materials.

High resolution structural, chemical, and electrical characterizations revealed that the overall electronic properties of $\mathrm{TMD}_{\mathrm{S}}$ are ultimately determined by peculiar nanoscale defects/inhomogeneities (e.g., point or complex defects, thickness fluctuations, grain boundaries, wrinkles, etc.), which can be intrinsic of these materials or dependent on the growth method, on the substrates and on the device fabrication processes. In this context, electrical atomic force microscopy methods, such as conductive atomic force microscopy (C-AFM), scanning capacitance microscopy (SCM), Kelvin probe force microscopy (KPFM), and scanning microwave impedance microscopy (SMIM) proved to be essential tools to understand the nature and electrical activity of defects/nonuniformities in 2D materials, and more specifically in TMDs [34-39]. In particular, C-AFM allows performing a high-resolution current mapping and local current-voltage (I-V) characterization at the nanoscale [40-43]. Hence, it is the method of choice to investigate the mechanisms of current injection from contacts to $2 \mathrm{D}$ materials [44,45], the lateral homogeneity of conductivity of 2D semimetals (graphene) [46-48], and semiconductors (TMDs) [44], as well as the transversal current transport across thin dielectric films [49,50] or 2D insulators (such as h-BN) [51-53]. Furthermore, C-AFM allows investigating the vertical current injection across the van der Waals heterostructures of 2D materials [12] or their heterojunctions with bulk semiconductors [19,54-56]. Figure 1 schematically illustrates these possible applications of C-AFM in the characterization of 2D materials and heterostructures.

(a)

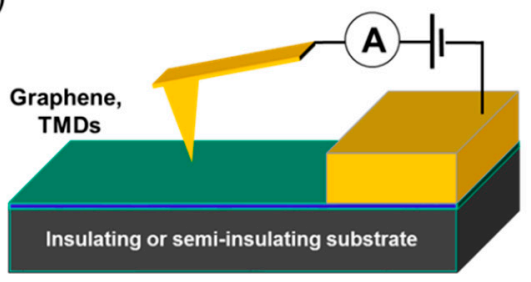

(c)

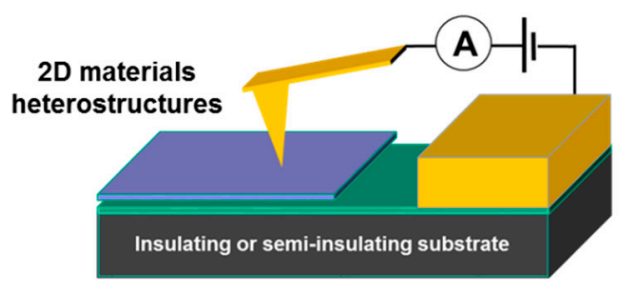

(b)

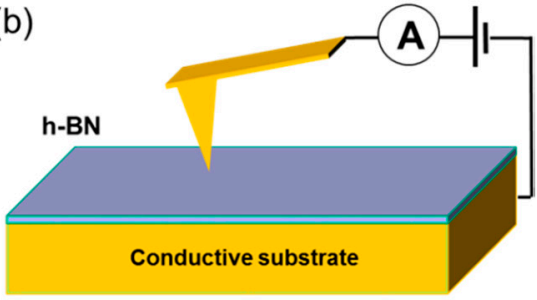

(d)

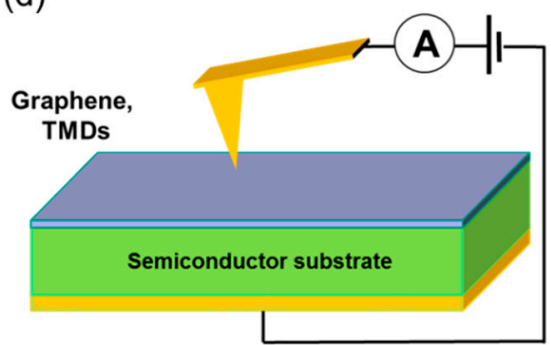

Figure 1. Schematic illustration of conductive atomic force microscopy (C-AFM) applications in two dimensional (2D) materials characterizations. (a) Lateral current transport in semi-metallic graphene or semiconducting TMDs. (b) Tunneling current through insulating hexagonal boron nitride (h-BN). (c) Current transport in 2D materials vertical (or lateral) heterostructures. (d) Current injection in the heterojunctions of 2D materials (graphene or TMDs) with bulk semiconductors.

This paper reviews some relevant applications of the C-AFM technique to the investigation of nanoscale transport properties in TMDs, discussing the implications of the local phenomena in the overall behavior of devices fabricated on these materials.

Section 2 includes recent studies of the current injection mechanisms at the metal/TMDs junctions. Nanoscale resolution current spectroscopy and mapping by C-AFM provided information on the 
Schottky barrier height (SBH) uniformity and shed light on the mechanisms responsible for the Fermi level pinning commonly observed at the metal/TMDs interface. The application of this approach to nanoscale mapping of the $\mathrm{SBH}$ distribution in $\mathrm{MoS}_{2}$ thin films subjected to oxygen plasma prefunctionalization is illustrated, and the practical implications of these results in the realization of ambipolar $\mathrm{MoS}_{2}$ transistors are also demonstrated.

Section 3 presents experiments on local conductivity mapping in monolayer $\mathrm{MoS}_{2}$ grown by CVD, providing a direct evidence of the role of grain boundaries (GBs) between $\mathrm{MoS}_{2}$ domains on the overall current transport in these layers.

Finally, Section 4 presents C-AFM studies of the current transport in TMD-based heterostructures, including lateral heterojunctions present in TMDs alloys, and vertical van der Waals heterojunctions formed by the stacking of different TMDs and of TMDs with bulk semiconductors.

\section{Schottky Barrier Height Mapping at Metal/TMDs Junction}

The lack of out-of-plane bonds in TMDs initially led to the expectation that the Schottky barrier at the interface between metal contacts and TMDs would approach the Schottky-Mott limit of weak pinning [57]. However, contrary to this expectation, experiments showed the opposite behavior, i.e., the SBH for most semiconducting TMDs is only weakly dependent on the metal work function, and this effect has been attributed to a Fermi level pinning [58-61]. Clearly, this phenomenon has strong implications on TMDs-based transistors, as the current injection/extraction from the source/drain contacts rules the overall device behavior [62]. As an example, in the case of $\mathrm{MoS}_{2}$ thin films (produced either by exfoliation or by CVD) most of the elementary metals exhibit a Fermi level pinning close to the $\mathrm{MoS}_{2}$ conduction band, resulting in SBH values for electrons injection ranging from 20-60 meV for low work function metals ( $\mathrm{Sc}$, Ti, etc.) to $150-250 \mathrm{meV}$ for high work function ones ( $\mathrm{Ni}, \mathrm{Pt}$, etc.) [58]. As a matter of fact, the low SBH for electrons translates into a high SBH for holes. Hence, n-type FETs with an electron accumulation channel can be easily obtained with unintentionally n-type doped $\mathrm{MoS}_{2}$, whereas the fabrication of $\mathrm{MoS}_{2}$ FETs with the complementary p-type behavior is challenging, due to the difficulty to inject holes in the inversion channel [63,64]. Contrary to the case of $\mathrm{MoS}_{2}$, a p-type

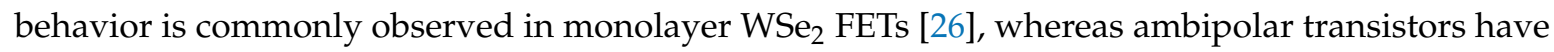
been demonstrated using a few layers of $\mathrm{MoTe}_{2}$ [65]. In spite of these differences, the electrical behavior of TMDs-based FETs is generally dominated by the Schottky barrier of source and drain contacts.

The origin of the Fermi level pinning at the junction between metals and TMDs is currently an object of debate. According to the commonly accepted models of Fermi level pinning at metal/semiconductor interfaces, this effect can be ascribed either to metal-induced gap states (MIGS) [66] or to the disorder induced gap states (DIGS) [67]. In the DIGS model, the Fermi level pinning is due to states in the electronic structure of the host semiconductor associated to defects in its crystalline structure. On the other hand, the MIGS model predicts that the states in the gap are induced by the interaction with the metal, i.e., the metal dominates over the substrate in pinning the Fermi level. Recently, ab-initio simulations of defect-free TMDs showed that the metal/TMD junctions largely follow the MIGS model, similar to three-dimensional semiconductors despite their different structure and bonding [59]. In this context, Fermi level depinning by the insertion of an ultra-thin tunnel barrier (such as monolayer h-BN) between contacts and $\mathrm{MoS}_{2}$ has been recently reported [30]. The main function of this barrier layer is increasing the physical separation between the $\mathrm{MoS}_{2}$ and the contact electrode, thereby minimizing the metal/MoS 2 interfacial interaction responsible for the creation of MIGS. On the other hand, early nanoscale electrical investigations (based on scanning tunneling microscopy) highlighted the possible role of the defects present at the $\mathrm{MoS}_{2}$ surface in the Fermi level pinning phenomenon [68].

As a matter of fact, $\mathrm{SBH}$ mapping with nanoscale resolution is required to disentangle the effect of surface (or near surface) defects in the TMD materials from that of metal-induced gap states. To this purpose, the conductive tip of C-AFM has been employed as a nanoscopic metal electrode to record local I-V characteristics on the TMDs surface, from which the SBH was quantitatively evaluated $[44,45,69]$. Contacting the TMD surface with a sliding metal tip presents the additional advantage of excluding 
eventual reactions that have been reported at the metal/TMD interface (for some metal species) when the contact is fabricated by evaporation or sputtering [70,71]. In this way, the effect of the interaction with a metal electrode in close proximity to the TMD surface can be investigated. In the following subsection, recent studies of the Fermi level pinning in TMDs using C-AFM are discussed.

\subsection{C-AFM Investigations of Fermi Level Pinning in TMDs}

The C-AFM technique was firstly employed in the current-voltage (I-V) spectroscopy mode to investigate the current injection to the surface of multilayer $\mathrm{MoS}_{2}$ exfoliated on a $\mathrm{SiO}_{2}$ substrate [44]. Local I-V measurements were performed under ambient conditions using a Pt coated Si tip (curvature radius $r_{\text {tip }} \approx 10 \mathrm{~nm}$ ) connected to a high sensitivity current amplifier, while a bias was applied to a macroscopic front contact, as schematically illustrated in Figure 2a. Figure $2 \mathrm{~b}$ shows a set of $25 I-V_{\text {tip }}$ curves (with $V_{\text {tip }}$ as the bias referred to the tip) measured on the $\mathrm{MoS}_{2}$ surface on a $0.5 \times 0.5 \mu \mathrm{m}$ area. All the curves are asymmetric from a positive (forward) to negative (reverse) bias, indicating a Schottky behavior of the Pt/ $\mathrm{MoS}_{2}$ contact. A representative forward $I-V_{\text {tip }}$ characteristic from this array of measurements is reported in Figure 2c. In the semilog-plot, a linear increase of the current over more than two decades (from $1 \times 10^{-10}$ to $5 \times 10^{-8} \mathrm{~A}$ ) is observed, followed by a saturation. The SBH $\left(\Phi_{B}\right)$ and the ideality factor $(n)$ for the nanoscopic $\mathrm{Pt} / \mathrm{MoS}_{2}$ contact were evaluated by fitting the low voltage region of the forward $I-V_{\text {tip }}$ curve with the thermionic emission law:

$$
I=A A^{*} T^{2} \exp \left(-\frac{q \Phi_{B}}{k_{B} T}\right) \exp \left(\frac{q V_{t i p}}{n k_{B} T}\right)
$$

where $q$ is the electron charge, $k_{B}$ is the Boltzmann constant, $T$ is the absolute temperature $(T=300 \mathrm{~K})$, $A=\pi r_{\text {tip }}{ }^{2}$ is the tip contact area, and $A^{*}$ is the Richardson constant of multilayer $\mathrm{MoS}_{2}$ [44]. In particular, $\Phi_{B}=307 \mathrm{meV}$ and $n=1.61$ were determined from the intercept and the slope of the linear fit of the $\ln (I)-V_{\text {tip }}$ characteristic for $V_{\text {tip }}<0.3 \mathrm{~V}$. The current saturation observed on the semilog-scale at higher bias values is due to a series resistance contribution $R$.

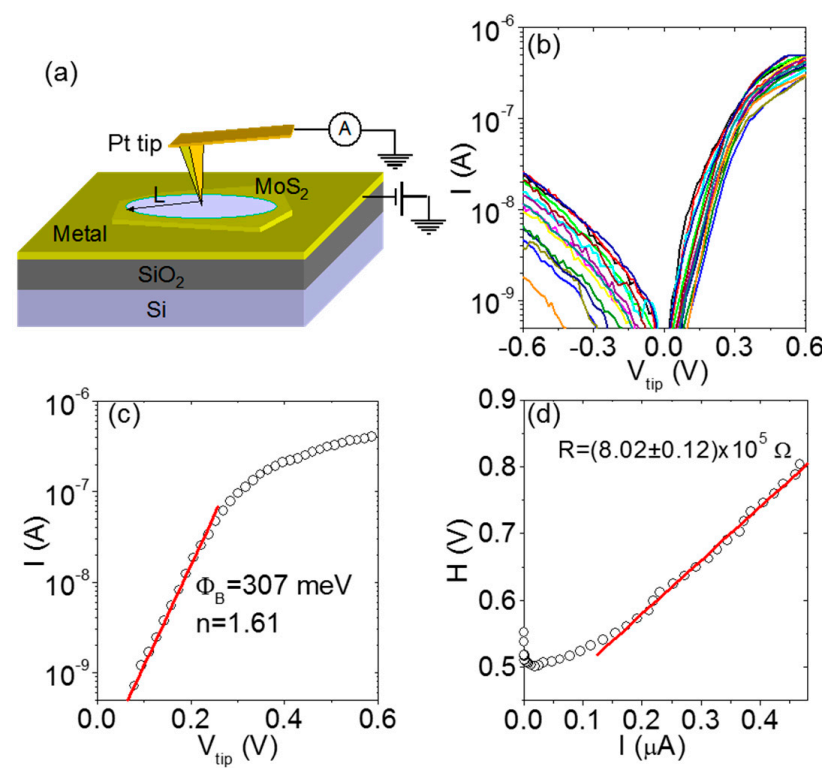

Figure 2. (a) Schematic of the experimental setup for C-AFM measurements on $\mathrm{MoS}_{2}$. (b) Set of 25 I- $V_{\text {tip }}$ characteristics measured on a $500 \times 500 \mathrm{~nm}$ array of tip positions with $\sim 100 \mathrm{~nm}$ spacing on $\mathrm{MoS}_{2}$. (c) Representative forward bias $I-V_{\text {tip }}$ characteristic from this set of measurements and fit with the thermionic emission law to extract the Schottky barrier height (SBH) and ideality factor. (d) The $H$ function plot for the determination of the series resistance $R$. Figures adapted with permission from Reference [44], copyright from the American Physical Society 2015. 
Since the downward curvature in the high voltage region of the $I-V_{\text {tip }}$ curves depends both on $n$ and $R$, the Cheung's method [72] was applied to evaluate the $R$ contribution. In this method, the function $H$ is defined as $H=V_{t i p} n k_{B} T / q \ln \left[I /\left(A A^{*} T^{2}\right)\right]$, which depends on $I$ as $H=n \Phi_{B}+I R$. Figure $2 \mathrm{~d}$ shows a plot of $H$ vs. $I$ obtained from the forward bias $I-V_{\text {tip }}$ characteristic in Figure $2 \mathrm{c}$. $R=(8.02 \pm 0.12) \times 10^{5} \Omega$ was evaluated by the slope of the linear fit for current values larger than $0.1 \mu \mathrm{A}$. The $\mathrm{R}$ value is mainly due to the spreading resistance $R_{\mathrm{spr}}$, associated to current spreading into multilayer $\mathrm{MoS}_{2}$ from the nanoscale contact. $R_{\mathrm{spr}}$ is related to the local resistivity $\rho_{\text {loc }}$ of $\mathrm{MoS}_{2}$ under the tip as $R_{\text {spr }}=\rho_{\text {loc }} /\left(4 \mathrm{r}_{\text {tip }}\right)$, being $\mathrm{r}_{\text {tip }}$ the contact radius. Hence, the local resistivity $\rho_{\text {loc }}$ could be estimated from the $R$ values measured at each tip position. By performing the same analysis on the full set of $I-V_{\text {tip }}$ characteristics of Figure $2 \mathrm{~b}$, the distributions of the local SBHs, ideality factors, and resistivity values at the different tip positions on $\mathrm{MoS}_{2}$ were determined. The histograms of the $\Phi_{B}, n$, and $\rho_{\text {loc }}$ values are reported in Figure $3 \mathrm{a}-\mathrm{c}$, respectively.
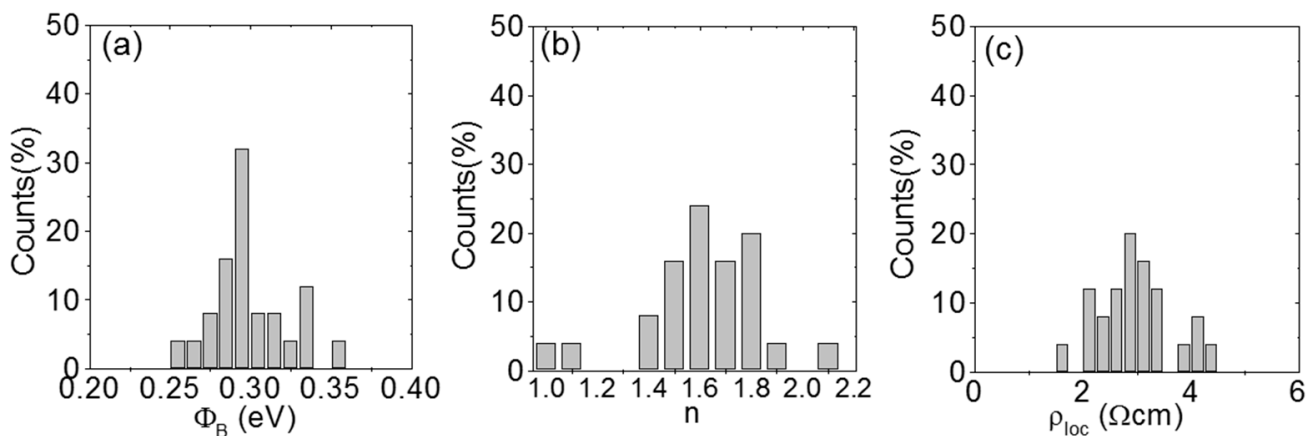

Figure 3. Histograms of the local Schottky barrier height $\Phi_{B}(\mathbf{a})$, ideality factor $n(\mathbf{b})$, and resistivity $\rho_{\text {loc }}(\mathbf{c})$ extracted from the array of $I-V_{\text {tip }}$ characteristics in Figure $2 \mathrm{~b}$. Figures adapted with permission from Reference [44], copyright from the American Physical Society 2015.

The distribution in Figure 3 a shows an average SBH of $300 \mathrm{meV}$ with a standard deviation of $24 \mathrm{meV}$. This average value is in good agreement with the SBH evaluated for deposited Pt contacts on multilayer $\mathrm{MoS}_{2}$ [58]. According to the Schottky-Mott theory, the ideal SBH value for a metal/semiconductor contact without Fermi level pinning is expressed as:

$$
\Phi_{B}=W_{M}-\chi
$$

where $W_{M}$ is the metal work function and $\chi$ is the semiconductor electron affinity. In the specific case of $\mathrm{Pt}$ and multilayer $\mathrm{MoS}_{2}, \Phi_{\mathrm{M}} \approx 5.4 \mathrm{eV}$ and $\chi \approx 4.1 \mathrm{eV}$, result in an ideal $\Phi_{B} \approx 1.3 \mathrm{eV}$. Clearly, the experimental SBH values are much lower than the ideal one. The histogram in Figure $3 \mathrm{~b}$ shows that $n$ is close to unity only on $\sim 10 \%$ of the investigated $\mathrm{MoS}_{2}$ area, whereas the average value of $n$ is 1.60 with a standard deviation of 0.23 . Generally, the deviation of $n$ from unity indicates that the current transport is not perfectly described by the thermionic emission theory and can be ascribed to the presence of surface states [73]. Finally, an average resistivity of $2.99 \Omega \mathrm{cm}$ with a standard deviation of $0.68 \Omega \mathrm{cm}$ was estimated from the distribution in Figure 3c. The lateral variations of $\rho_{\text {loc }}$ can be ascribed to inhomogeneities in the carrier concentration and/or in the carrier mobility of $\mathrm{MoS}_{2}$.

Since these C-AFM analyses were carried out under ambient conditions, the measured $\mathrm{SBH}$ distribution can be affected by the presence of a water meniscus under the tip, which is known to cause a degradation of the lateral resolution. In this context, performing C-AFM under a high vacuum or within an environmental chamber has been shown to allow current mapping and spectroscopy with a greatly improved resolution [74].

Recently, high resolution current mapping by an environmental chamber C-AFM, combined with atomic resolution scanning tunneling microscopy (STM), provided further insight on the nature of defects responsible for the Fermi level pinning in $\mathrm{MoS}_{2}$ [45] and other TMDs (MoSe $2, \mathrm{MoTe}_{2}$, 
$\mathrm{WS}_{2}$, and $\mathrm{WSe}_{2}$ ) [75]. Figure 4a shows a lateral force microscopy (LFM) and the corresponding AFM topographic image (inset) of a freshly cleaved $\mathrm{MoS}_{2}$ sample, showing a smooth surface in both images [45]. On the other hand, the simultaneously recorded C-AFM map (Figure 4b) shows a laterally inhomogeneous current injection through the nanoscopic contact between the conductive tip (made of boron doped diamond) and the $\mathrm{MoS}_{2}$ surface. Multiple dark circular features (with radii ranging between 3 and $4 \mathrm{~nm}$ ) are present in this image, with a higher current than the surrounding areas, indicating a lower tip/ $\mathrm{MoS}_{2}$ contact resistance. The surface density of these features was found in the range between $10^{10}$ and $10^{11} \mathrm{~cm}^{-2}$, depending on the sample and location. Atomic resolution scanning tunneling microscopy (STM) analyses of the $\mathrm{MoS}_{2}$ surface were also carried out to understand the nature of the high current spots in the C-AFM images. The STM maps revealed the presence of characteristic features appearing as circular depressions on which the $\mathrm{MoS}_{2}$ lattice is superimposed (see Figure $4 \mathrm{c}$ ). The typical lateral size of these depressions was up to $5 \mathrm{~nm}$ and their areal density was $(8 \pm 3) \times 10^{10} \mathrm{~cm}^{-2}$, similar to the density of conductive features in the C-AFM images. The absence of such depressions in the topographic and LFM images (Figure 4a) suggested that the observed features in the STM maps are electronic in nature and induced by subsurface defects. They were associated to Mo-vacancies or Mo-substitutional defects, located below the outermost S layer [45]. In addition to these subsurface defects, sulfur vacancies (see Figure $4 \mathrm{~d}$ ) were found to be ubiquitously present in the topmost $S$ layer of $\mathrm{MoS}_{2}$, with an areal density of $(7 \pm 4) \times 10^{12} \mathrm{~cm}^{-2}$. No specific features corresponding to these point defects were observed in the C-AFM map.
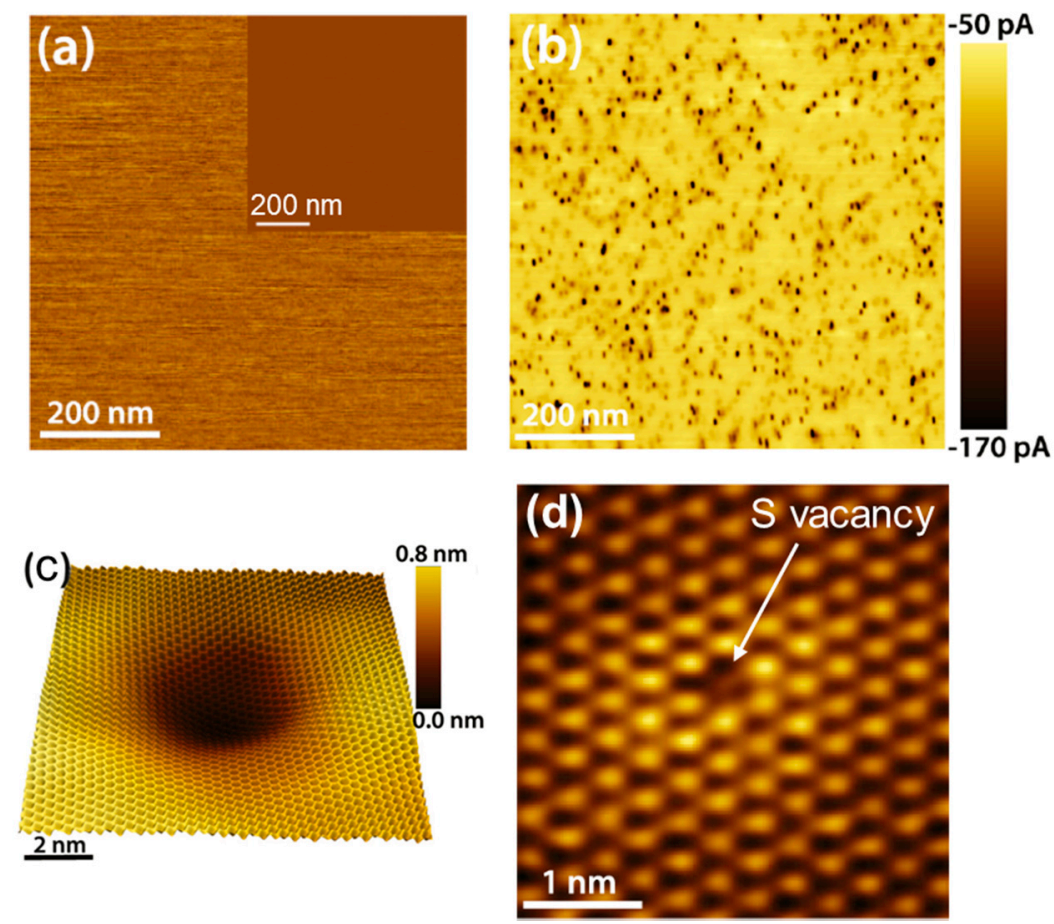

Figure 4. (a) Lateral force microscopy (LFM) and topography (insert) image of the $\mathrm{MoS}_{2}$ surface. (b) Simultaneously recorded C-AFM image. (c) Scanning tunneling microscopy (STM) image of a subsurface defect, corresponding to one of the high current spots in the panel (b). (d) STM image of a sulfur vacancy in the outermost sulfur layer. Images adapted with permission from Reference [45], copyright from the American Chemical Society 2017.

Figure 5a shows a map of the SBH distribution extracted from an array of $128 \times 128 \mathrm{I}-\mathrm{V}$ curves collected by the conductive diamond tip on the freshly cleaved $\mathrm{MoS}_{2}$ surface. More specifically, the local $\Phi_{B}$ values were obtained by fitting the low forward bias region of the individual I-V curves with the thermionic emission model, as already discussed in Figure 2c. This high resolution SBH map showed minimum $\Phi_{B}$ values of $\sim 0.3 \mathrm{eV}$ corresponding to the subsurface defect regions, whereas a 
nearly constant SBH of $\sim 0.53 \mathrm{eV}$ was observed in the surrounding areas. As a matter of fact, these SBH values are much smaller than the ideal one $(\sim 1 \mathrm{eV})$ predicted by the Schottky-Mott Equation (2) in the specific case of a boron doped diamond tip (work function $\Phi_{\mathrm{M}}=5.1 \mathrm{eV}$ ) in contact with $\mathrm{MoS}_{2}$. Hence, even in the regions without the subsurface defects, a Fermi level pinning occurs, although less intense than on defects. The authors excluded that this Fermi level pinning can be due to the sulfur vacancies, and ascribed this effect to gap states induced by the interaction of the metal tip with $\mathrm{MoS}_{2}$, as indicated also by ab-initio calculations [59]. Clearly, the high density of low $\Phi_{B} \approx 0.3 \mathrm{eV}$ patches (separated by a distance of $\sim 10 \mathrm{~nm}$ from each other) dominates over the background with higher $\Phi_{B} \approx 0.53 \mathrm{eV}$ and determines the effective SBH. Based on these considerations, the results of this high resolution C-AFM investigation are in agreement with those of the C-AFM study in [44], as well as with SBH measurements on macroscopic contacts [58].

(a)
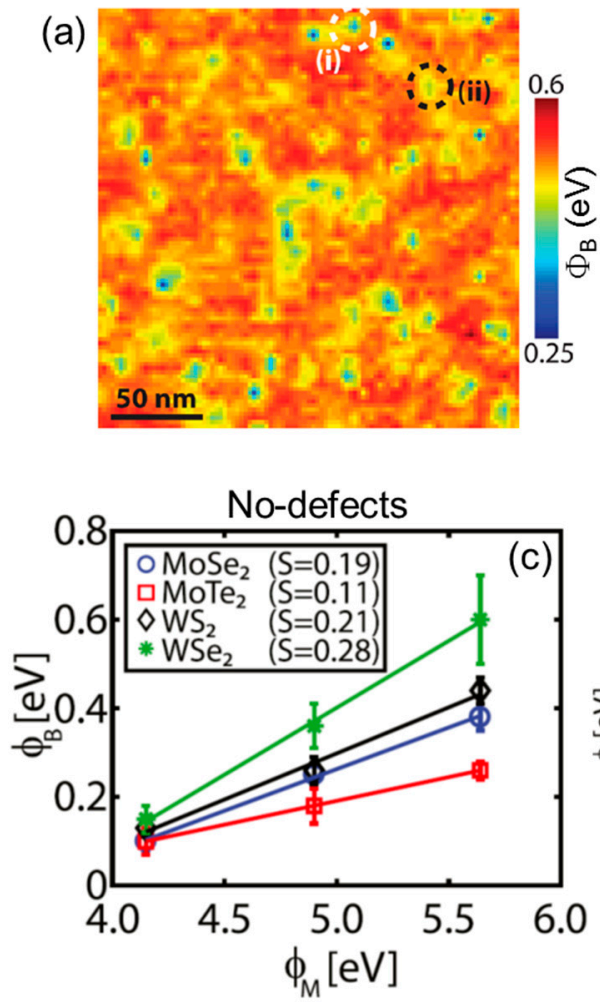
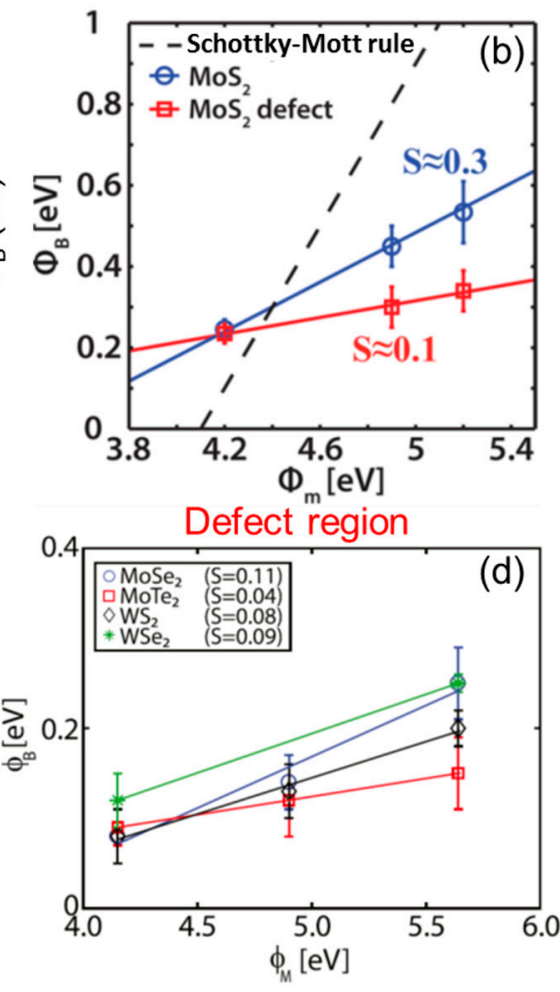

Figure 5. (a) Schottky barrier height (SBH) map on the $\mathrm{MoS}_{2}$ surface obtained by fitting an array of $128 \times 128$ local current-voltage (I-V) curves with the thermionic emission model. (b) SBH for the defect-free $\mathrm{MoS}_{2}$ areas (blue) and on subsurface defects (red) vs. the metal tip work function $\left(\Phi_{\mathrm{M}}\right)$. The pinning factor $S$ was evaluated as the slope of the linear fit. The dotted line is the standard Schottky-Mott rule. The SBH of different TMDs $\left(\mathrm{MoSe}_{2}, \mathrm{MoTe}_{2}, \mathrm{WS}_{2}, \mathrm{WSe}_{2}\right)$ for defect-free (c) and defect regions (d) vs. the metal tip work function. Panels (a) and (b) adapted with permission from Reference [45], copyright from the Chemical Society 2017. Panels (c) and (b) adapted with permission from Reference [75], copyright from the American Chemical Society 2019.

To get a more complete description of the Fermi level pinning effect, the dependence of the local SBH on the metal tip work function was also investigated [45]. Figure $5 b$ shows a plot of the experimental $\Phi_{B}$ values as a function of the metal tip work function both on the defect-free $\mathrm{MoS}_{2}$ areas and on the subsurface defect regions. In addition to the conductive diamond tip $\left(\Phi_{\mathrm{M}}=5.1 \mathrm{eV}\right)$, a PtSi tip $\left(\Phi_{\mathrm{M}} \approx 4.9 \mathrm{eV}\right)$ and a highly n-type doped Si tip $\left(\Phi_{\mathrm{M}} \approx 4.1-4.2 \mathrm{eV}\right)$ were used in this experiment. The pinning factor $S=\mathrm{d} \Phi_{B} / \mathrm{d} \Phi_{\mathrm{M}}$ was evaluated as the slope of the linear fit of these data. The obtained values for $S$ are $\sim 0.3$ and $\sim 0.1$ for the $M_{o} S_{2}$ regions without defects and for the regions with subsurface defects, respectively. The observed pinning factor of the defect-free $\mathrm{MoS}_{2}$ areas was found to be 
consistent with the theoretically predicted values for pristine $\mathrm{MoS}_{2}$ considering gap states induced exclusively by the metal/TMD interaction $[60,61]$. On the other hand, the measured pinning factor on the defect sites matches well with the experimentally obtained values on metal/MoS 2 contacts $[58,60,76]$, confirming the dominant role of these defects on the overall contact behavior.

This kind of investigation was also extended to other TMDs, i.e., $\mathrm{MoSe}_{2}, \mathrm{MoTe}_{2}, \mathrm{WS}_{2}$, and $\mathrm{WSe}_{2}$ [75]. Figure 5c,d shows the SBH values for the different TMDs evaluated in the defect-free (c) and in the defect regions (d) as a function of the metal tip work function. A similar behavior in the case of $\mathrm{MoS}_{2}$ is observed also for these TMDs, i.e., the presence of Fermi level pinning in the defect-free regions, ascribed to gap states induced by the metal tip, and an even stronger pinning (30-40\% increase) on defect regions due to defects induced gap states.

\subsection{Nanoscale Mapping of $\mathrm{MoS}_{2}$ Schottky Barrier Tuned by Oxygen Plasma Treatments}

As previously discussed, the Fermi level pinning at the metal/TMDs interface has important implications in electronic device performances, since the resulting Schottky barrier ultimately rules the device on-resistance, as well as the type of carriers (n-type or p-type) that can be injected in the channel of TMD-based transistors. In the specific case of $\mathrm{MoS}_{2}$, which is an unintentionally n-type doped semiconductor, the Fermi level pinning close to the conduction band results in a relatively small $\mathrm{SBH}$ for electron injection and in a high SBH for holes injection. Hence, $\mathrm{MoS}_{2}$ transistors commonly exhibit an n-type behavior associated with the formation of an electron accumulation channel for positive gate bias above the threshold voltage, whereas the observation of a p-type behavior related to the hole inversion channel at a sufficiently high negative bias is typically precluded. Clearly, this represents an obstacle for the realization of a complementary MOS technology all based on $\mathrm{MoS}_{2}$.

Recently, soft $\mathrm{O}_{2}$ plasma functionalization revealed an effective method to finely tailor the $\mathrm{SBH}$ of multilayer $\mathrm{MoS}_{2}$, and this approach was exploited to demonstrate $\mathrm{MoS}_{2}$ field effect transistors with ambipolar (i.e., both n-and p-type) behavior [29]. In this context, nanoscale electrical analyses by C-AFM showed how the $\mathrm{SBH}$ of $\mathrm{MoS}_{2}$ can be tuned by increasing the plasma exposure time [29]. Figure 6a,d reports the SBH map and the histogram of the $\Phi_{B}$ values measured on pristine $\mathrm{MoS}_{2}$, showing a narrow SBH distribution. Figure $6 \mathrm{~b}$ reports the SBH map after the $300 \mathrm{~s}$ soft plasma treatment, which results in a broader $\mathrm{SBH}$ distribution, with $\Phi_{\mathrm{B}}$ ranging from 0.21 to $0.58 \mathrm{eV}$, as shown by the histogram of the $\Phi_{\mathrm{B}}$ values (see Figure 6e). Finally, Figure 6c shows the SBH map after the $600 \mathrm{~s}$ soft plasma treatment. In this case, the $\mathrm{SBH}$ distribution extends from $\sim 0.2$ to $\sim 0.9 \mathrm{eV}$ (see Figure $6 \mathrm{f}$ ). Hence, it includes both regions with a low barrier for electrons and regions with a low barrier for holes, being the Schottky barrier for holes $\Phi_{\mathrm{B}, \mathrm{h}}=\mathrm{E}_{\mathrm{g}}-\Phi_{\mathrm{B}}$, with $\mathrm{E}_{\mathrm{g}}$ as the bandgap of multilayer $\mathrm{MoS}_{2}$.

Hence, starting from a narrow distribution of low SBHs for electrons in the case of pristine $\mathrm{MoS}_{2}$, the SBH map was modified after a $600 \mathrm{~s} \mathrm{O}_{2}$ plasma treatment into a broad distribution formed by nanometric patches with low SBH for holes in a background with low SBH for electrons. These $\mathrm{SBH}$ inhomogeneities in the $\mathrm{O}_{2}$ plasma treated samples were associated to lateral variations of the incorporated oxygen concentration in the $\mathrm{MoS}_{2}$ surface region [29]. Back-gated FETs were fabricated with Ni source and drain contacts deposited on pristine $\mathrm{MoS}_{2}$ (see schematic in Figure 7a) or on areas selectively exposed to $\mathrm{O}_{2}$ plasma functionalization for $600 \mathrm{~s}$ (see schematic in Figure 7c). Figure 7b shows the transfer characteristics $\mathrm{I}_{\mathrm{D}}-\mathrm{V}_{\mathrm{G}}$ for different drain bias values $\left(\mathrm{V}_{\mathrm{DS}}=1,2\right.$, and $5 \mathrm{~V}$ ) measured on a FET with channel length of $\mathrm{L}=10 \mu \mathrm{m}$ fabricated on a pristine $\mathrm{MoS}_{2}$ flake. The n-type transistor behavior typically reported for $\mathrm{MoS}_{2}$ FETs can be observed, with a monotonic increase of $\mathrm{I}_{\mathrm{D}}$ over more than five decades in the considered gate bias range. Figure $7 d$ shows the transfer characteristics $I_{D}-V_{G}$ (for $\mathrm{V}_{\mathrm{DS}}=1,2$, and $5 \mathrm{~V}$ ) of a $\mathrm{MoS}_{2}$ transistor with the same channel length and thickness, but with the source and drain contacts deposited on plasma $\mathrm{O}_{2}$ functionalized regions. For $\mathrm{V}_{\mathrm{DS}}=1$ and $2 \mathrm{~V}$, the transfer characteristics exhibit a pronounced p-type behavior, with the hole current branch (for $V_{G}<0$ ) significantly higher than the electron current branch (for $V_{G}<0$ ), whereas for $V_{D S}=5 \mathrm{~V}$ an ambipolar behavior is observed, with the $\mathrm{I}_{\mathrm{on}} / \mathrm{I}_{\text {off }}$ current ratio of $\sim 10^{3}$ for both the electrons and holes branches. 

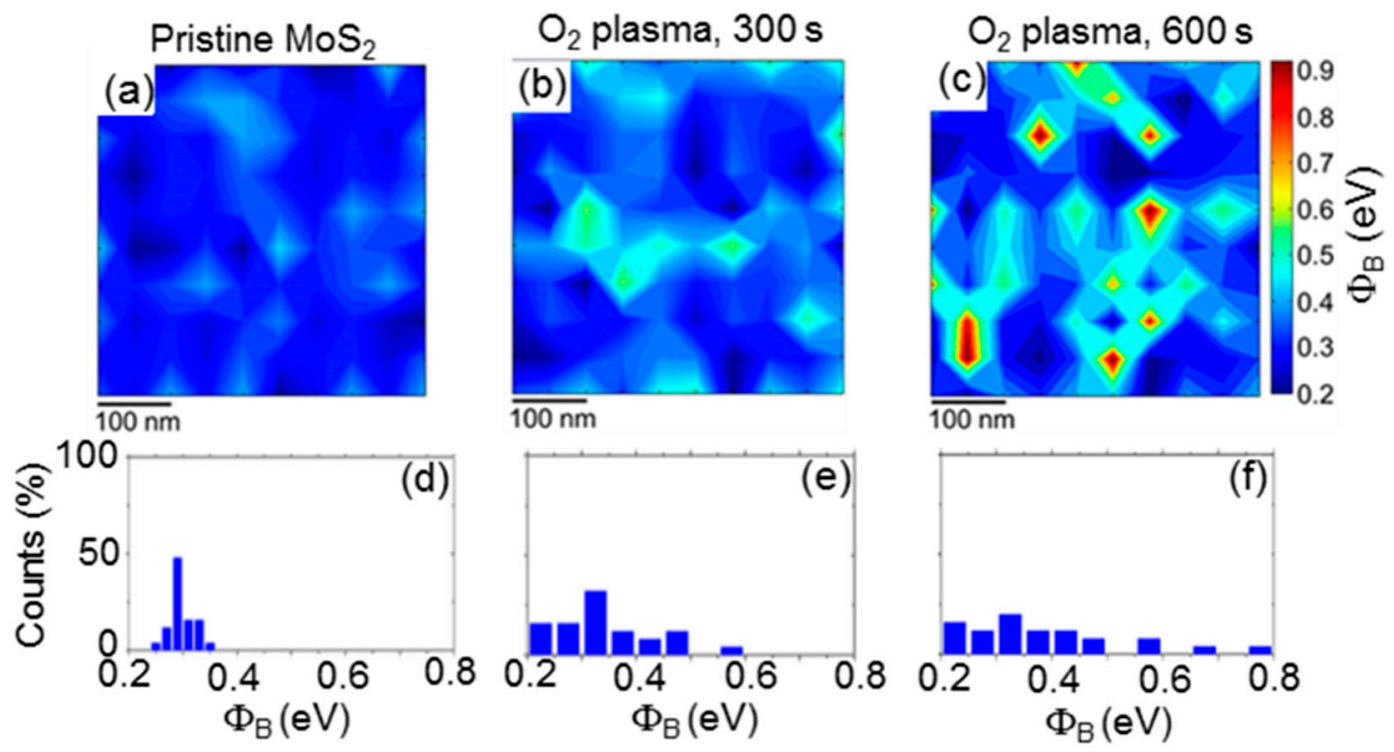

Figure 6. Schottky barrier height maps and histograms on pristine $\mathrm{MoS}_{2}(\mathbf{a}, \mathbf{d})$ and after $\mathrm{O}_{2}$ plasma for $300(\mathbf{b}, \mathbf{e})$ and $600 \mathrm{~s}(\mathbf{c}, \mathbf{f})$. Images adapted with permission from Reference [29], copyright from the American Chemical Society 2017.

(a)

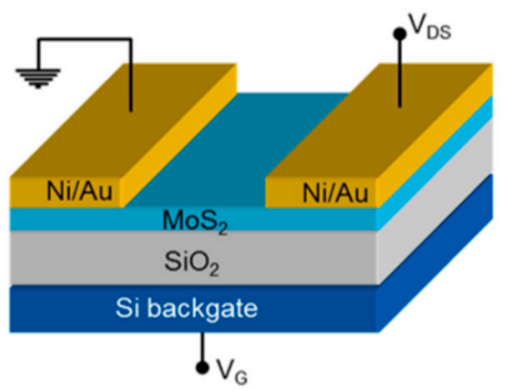

(c)

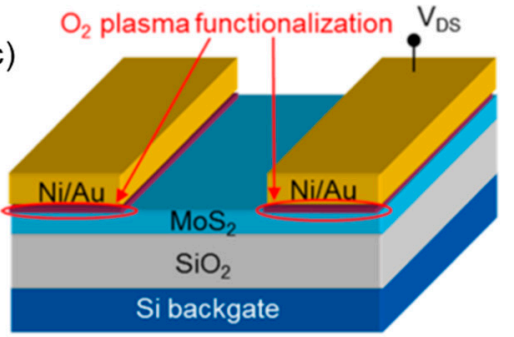

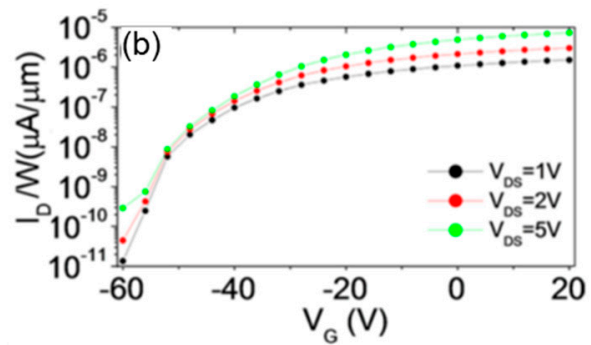

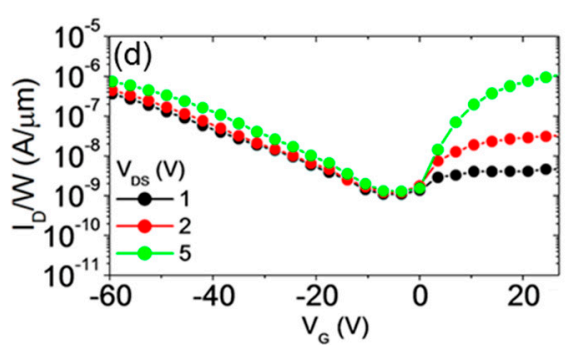

Figure 7. Schematic illustrations of back-gated field-effect transistors with source and drain contacts deposited on pristine $\mathrm{MoS}_{2}$ (a) or on areas selectively exposed to the $\mathrm{O}_{2}$ plasma for $600 \mathrm{~s}$ (c). $\mathrm{I}_{\mathrm{D}}-\mathrm{V}_{\mathrm{G}}$ characteristics for a pristine $\mathrm{MoS}_{2}$ transistor $(\mathbf{b})$ and for a transistor with $\mathrm{O}_{2}$ functionalized contact areas (d). Adapted with permission from Reference [29], copyright from the American Chemical Society 2017.

The ambipolar behavior in $\mathrm{O}_{2}$ functionalized FETs was explained by the coexistence of regions at low SBH for electrons and regions at low SBH for holes within the same source and drain contact areas, as demonstrated in the SBH map of Figure $6 c$. In particular, for a positively biased drain contact $\left(V_{D S}>0\right)$, the injection of electrons from the source to the accumulation channel (for $V_{G}>0$ ) occurs through regions with lower SBH for electrons, whereas the injection of holes from the drain to the inversion channel (for $\mathrm{V}_{\mathrm{G}}<0$ ) is allowed by the regions with low $\mathrm{SBH}$ for holes. The possibility to have both the $\mathrm{n}$ - and p-type current transport in a single device structure is an important step towards the implementation of a CMOS technology with $\mathrm{MoS}_{2}$. 


\section{Local Resistance Mapping at Grain Boundaries in CVD Grown $\mathrm{MoS}_{2}$}

The C-AFM experiments reviewed in the previous section demonstrated the enhanced current injection due to localized defects on the surface or near-surface region of multilayer TMDs [44,45,75]. These defects are responsible for the Fermi level pinning effect commonly observed at metal Schottky junctions with these semiconducting materials.

In addition to these localized defects, the presence of extended defects, such as grain boundaries (GBs), must be taken into account in the case of CVD grown TMDs. As an example, the CVD deposition of $\mathrm{MoS}_{2}$ on a commonly used $\mathrm{SiO}_{2} / \mathrm{Si}$ substrate typically results in the formation of a $2 \mathrm{D}$ polycrystalline material, composed by several domains with a triangular shape separated by GBs [77]. The structural and electrical properties of GBs have been the object of many experimental and theoretical investigations in the last years [78,79], due to their impact on the mobility of $\mathrm{MoS}_{2}$ field effect transistors and their role in peculiar extrinsic charge transport phenomena in $\mathrm{MoS}_{2}$ [80,81]. Recent investigations indicated that inter-domains scattering (due to GBs) can play a major role (as compared to the intra-domain scattering due to localized defects $[68,82,83])$ in the degradation of the $\mathrm{MoS}_{2}$ mobility, especially for certain misorientation angles between $\mathrm{MoS}_{2}$ domains [81]. To this purpose, complex transistors structures and modeling for electrical data interpretation have been employed to evaluate the impact of the specific GB configurations on the $\mathrm{MoS}_{2}$ channel mobility [81]. In this context, C-AFM has been recently employed as a powerful tool for direct probing of the electrical properties of $\mathrm{MoS}_{2}$ domains and GBs. As an example, the C-AFM analyses of a few layers of $\mathrm{MoS}_{2}$ grown by CVD on sapphire revealed a reduced current injection in the GBs, located in the topmost and in the buried $\mathrm{MoS}_{2}$ layers [84].

Nanoscale resolution current mapping by C-AFM has been also employed to evaluate the conductance drop associated with GBs in monolayer $\mathrm{MoS}_{2}$ grown by CVD onto a $\mathrm{SiO}_{2} / \mathrm{Si}$ substrate [85]. Figure 8a shows an optical microscopy of the as-grown domains, which exhibit typical triangular shapes and sizes up to $50 \mu \mathrm{m}$. The coalescence of $\mathrm{MoS}_{2}$ domains, resulting in the formation of GBs, can be deduced from this optical image. Figure $8 \mathrm{~b}$ illustrates the employed experimental configuration for C-AFM measurements, where the current is measured between the nanoscale contact formed by a PtIr tip and a macroscopic electrode partially covering some $\mathrm{MoS}_{2}$ domains. Figure $8 \mathrm{c}$,d shows the surface morphology and the corresponding current map measured with an applied bias $V_{\text {tip }}=1 \mathrm{~V}$ (referred to the tip) in the region indicated by a red box in Figure 8b. This area includes two $\mathrm{MoS}_{2}$ domains (indicated as D1 and D2) separated by a GB, with D1 directly connected to the macroscopic contact. The GB is clearly visible both in the morphology (as a sharp peak with $\sim 1 \mathrm{~nm}$ height) and in the current map. The current contrast is almost uniform within the individual domains, whereas a significant conductance drop can be observed from the domain D1 to the domain D2 separated by the GB. This is further elucidated by the two scan lines in Figure 8e,f, extracted from the morphology and current map, respectively. A reduction of the current by about a factor of 7 can be observed moving from D1 to D2, which is clearly associated to the resistance contribution of the GB. Furthermore, a dip in the current can be observed at the GB position. Noteworthy, the width of this current dip is $~ 150 \mathrm{~nm}$, much larger than the width of the peak in the height profile. This can be explained by a potential barrier associated to the GB, with the formation of depletion regions at the two sides of this barrier. This picture is consistent with the scenario proposed in Reference [81], where a potential barrier ranging from $\sim 0.2$ to $\sim 0.6 \mathrm{eV}$ was calculated, depending on the misorientation angle between adjacent $\mathrm{MoS}_{2}$ domains.

Hence, C-AFM represents a powerful tool to directly evaluate the impact of GBs in polycrystalline TMDs grown by CVD, without the need for fabricating ad hoc device structures. 
(a)

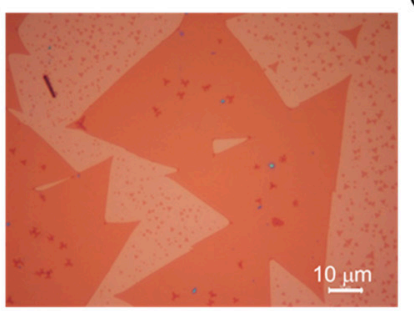

(b)

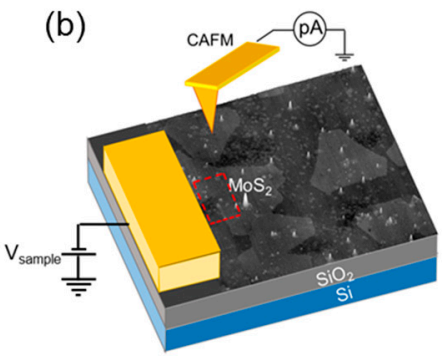

(c)

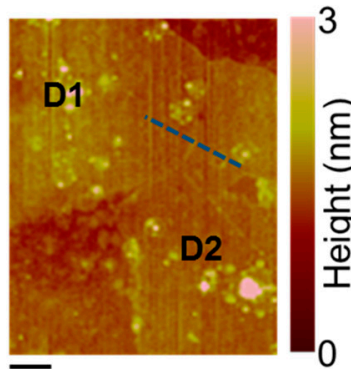

$\overline{1 \mu \mathrm{m}}$

(d)

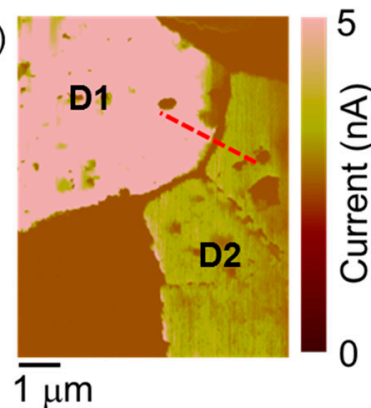

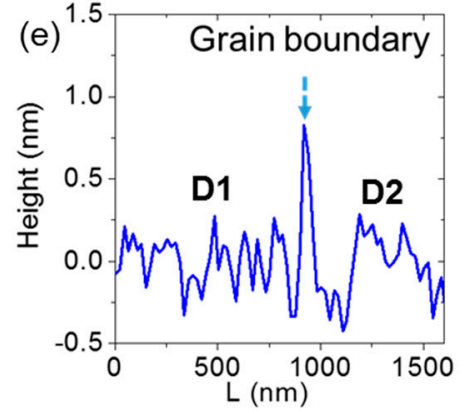

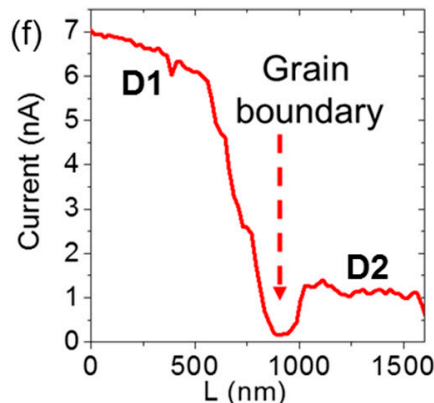

Figure 8. (a) Optical microscopy of CVD-grown monolayer $\mathrm{MoS}_{2}$ domains on a $\mathrm{SiO}_{2} / \mathrm{Si}$ substrate. (b) Illustration of the experimental setup for the local current measurement on $\mathrm{MoS}_{2}$ domains by C-AFM. (c) Morphology and (d) current map measured by C-AFM on a region with two MoS 2 domains (D1 and D2) separated by a grain boundary. The domain D1 is directly connected to the macroscopic contact. (e) Height and (f) current line-scans across the grain boundary. Images adapted with permission from Reference [85], copyright from Wiley 2020.

\section{Local Transport Properties in TMD Lateral and Vertical Heterojunctions}

\subsection{Lateral Heterojunctions of TMDs}

Due to their unique electronic properties, TMDs offer various solutions to realize lateral, i.e., in-plane, semiconductor heterojunctions. As an example, the significant bandgap variation from monolayer (1L) to bilayer (2L) of TMDs has been exploited as a simple way to create lateral heterostructures at the boundaries between $1 \mathrm{~L}$ and $2 \mathrm{~L}$ regions of the same material [86]. Another promising way to tune the TMD electronic structure (i.e., the band gap and the conduction/valence band energies) is the formation of alloys, obtained by mixing Mo with $\mathrm{W}$ and $\mathrm{S}$ with Se in a controlled way [87-89]. As an example, single layers of $\mathrm{Mo}_{\mathrm{x}} \mathrm{W}_{1-\mathrm{x}} \mathrm{Se}_{2}$ alloys have shown a tunable band gap, with the conduction/valence band levels depending on the exact ratio of Mo and W [90]. Although Mo and $\mathrm{W}$ exhibit a similar electronic structure, preferential segregation of Mo-rich and W-rich regions has been commonly observed during the growth process of the TMD alloys [91], resulting in the formation of one-dimensional (1D) lateral heterojunctions [92].

Recently, Bampoulis et al. [69] used C-AFM to characterize the conductivity of a $\mathrm{Mo}_{x} \mathrm{~W}_{1-x} \mathrm{Se}_{2}$ alloy at the nanoscale. They observed the segregation of Mo-rich and $\mathrm{W}$-rich domains and demonstrated that these different regions exhibit distinct SBHs values, reflecting the different band structures of $\mathrm{WSe}_{2}$ and $\mathrm{MoSe}_{2}$. An atomic resolution C-AFM map of a $\mathrm{Mo}_{x} \mathrm{~W}_{1-x} \mathrm{Se}_{2}$ alloy (with $\mathrm{x}=0.3$ ) is reported in Figure 9a, showing distinct differences in the conductivity between neighboring regions with a nanometer size (indicated with (i) and (ii)). To get further insight into this observation, a local I-V spectroscopy was carried out on these differently conductive regions. Figure $9 \mathrm{~b}$ shows a series of I-V characteristics recorded along the line indicated in the inset which depicts the boundary between the Mo-rich (more conductive) and W-rich regions (less conductive). The I-V curves appear to gradually change when moving from one region to the other. Figure $9 \mathrm{c}$ shows the SBH values along this line, extracted by thermionic emission fitting of the individual I-V curves in the forward bias regime. The transition 
to the smaller SBH (Mo-rich) regions happens gradually and on the length scale of about 3-4 nm. This gradual transition is due to band bending across the phase boundary. The Mo-rich and W-rich regions were expected to approach the electronic band diagram of the $\mathrm{MoSe}_{2}$ and $\mathrm{WSe}_{2}$ crystals, as schematically illustrated in Figure 9d. Based on this diagram, a type-II staggered gap heterojunction is expected when moving from a Mo-rich region to a W-rich region, and band bending occurs at the interface of the two regions. Finally, assuming that the Fermi level pins at the same energy level across the boundary, a conduction band offset (CBO) of $\sim 0.13 \mathrm{eV}$ was estimated from the SBH difference between the two regions.
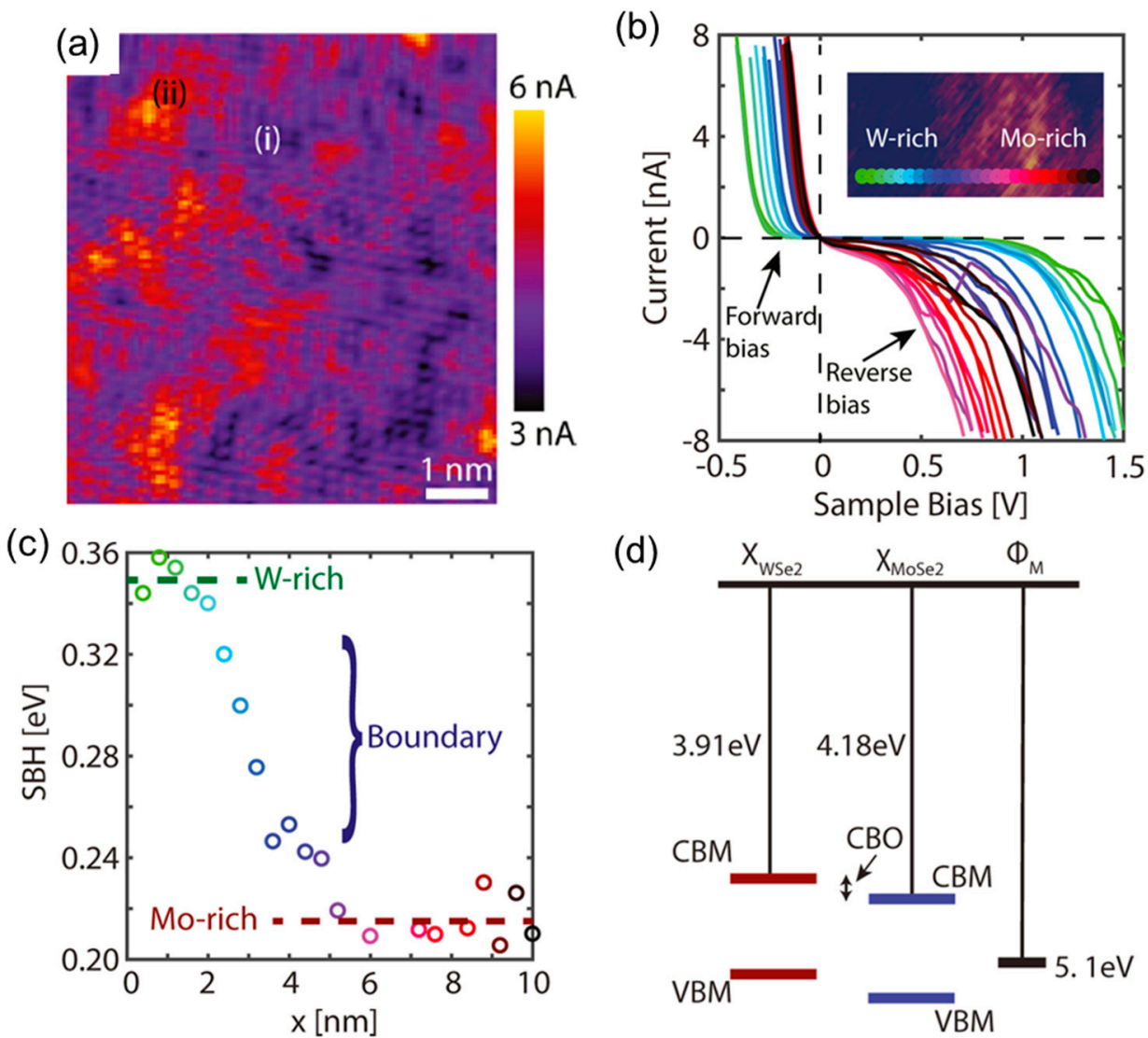

Figure 9. (a) C-AFM image of the surface of a $\mathrm{Mo}_{0.3} \mathrm{~W}_{0.7} \mathrm{Se}_{2}$ alloy, showing regions with different conductivities. (b) I-V characteristics across a W-rich/Mo-rich boundary (insert); the rainbow line indicates the positions where the I-V characteristics were recorded. (c) $\mathrm{SBH}$ as a function of the position along the line, with the color code corresponding to the exact I-V curves of panel (b). (d) Schematic band diagram of a $\mathrm{WSe}_{2} / \mathrm{MoSe}_{2}$ lateral heterojunction, illustrating the conduction band minima (CBM) and valence band maxima (VBM) of $\mathrm{WSe}_{2}$ and $\mathrm{MoSe}_{2}$ and the conduction band offset (CBO). Images adapted with permission from Reference [69], copyright from the American Chemical Society 2018.

\subsection{Vertical Heterostructures of TMDs}

In addition to the 1D lateral heterojunctions discussed in the previous paragraph, 2D semiconducting heterostructures obtained by vertical stacking of different TMDs are currently the object of increasing scientific interest, due to the wide range of potential applications in electronics and optoelectronics [10]. In particular, 2D heterostructures of TMDs with a type II band alignment, such as $\mathrm{MoS}_{2}$ and $\mathrm{WSe}_{2}$, offer the possibility to implement band-to-band tunneling diodes and transistors for ultra-low-power consumption logic applications.

Recently, Lin et al. [12] reported the growth of $\mathrm{MoS}_{2} / \mathrm{WSe}_{2}$ heterostructures onto epitaxial graphene $(\mathrm{EG})$ on $\mathrm{SiC}$ by sequentially depositing monolayers of the two TMDs employing oxide powder vaporization or metal-organic CVD. In particular, WSe 2 was first grown on EG at $950{ }^{\circ} \mathrm{C}$. 
Following this first growth step, the surface coverage of the WSe 2 on EG was typically $>60 \%$, with a lateral size of $2 \mu \mathrm{m}$ for $\mathrm{WSe}_{2}$ domains, as illustrated by the AFM image in Figure 10a. Subsequently, the growth of $\mathrm{MoS}_{2}$ on $\mathrm{WSe}_{2} / \mathrm{EG}$ was performed at $750{ }^{\circ} \mathrm{C}$. The $\mathrm{MoS}_{2}$ domains are smaller $(\sim 300 \mathrm{~nm})$ and typically grow on $\mathrm{WSe}_{2}$ starting from the domain's edges, as illustrated by the AFM image in Figure 10b. Given the small size of the $\mathrm{MoS}_{2} / \mathrm{WSe}_{2}$ areas, C-AFM was employed to investigate a vertical current transport across the heterostructure [12]. In particular, local I-V measurements through the $\mathrm{MoS}_{2} / \mathrm{WSe}_{2} / \mathrm{EG}$ and $\mathrm{WSe} / \mathrm{EG}$ heterostructures were carried out at room temperature according to the schematic in Figure 10c. Representative I-V curves collected on the two systems are reported in Figure $10 \mathrm{~d}$. The I-V characteristics of the $\mathrm{WSe}_{2} / \mathrm{EG}$ heterojunction exhibit a slight rectifying behavior, associated to the p-n junction between p-type $\mathrm{WSe}_{2}$ and n-type EG on SiC. On the other hand, the curves measured on the $\mathrm{MoS}_{2} / \mathrm{WSe} 2 / \mathrm{EG}$ show a current peak $\left(\right.$ at $\mathrm{V}_{\text {peak }} \approx 1.1 \mathrm{~V}$ ) followed by a valley (with a peak-to-valley current ratio of 1.9) and, finally, an exponential current increase. The negative-differential-resistance (i.e., the Esaki diode behavior) is a clear evidence of band-to-band-tunneling occurring at room temperature, indicating the formation of an ultra-sharp interface at the $\mathrm{MoS}_{2} / \mathrm{WSe}_{2} \mathrm{n}-\mathrm{p}$ junction.

(a)
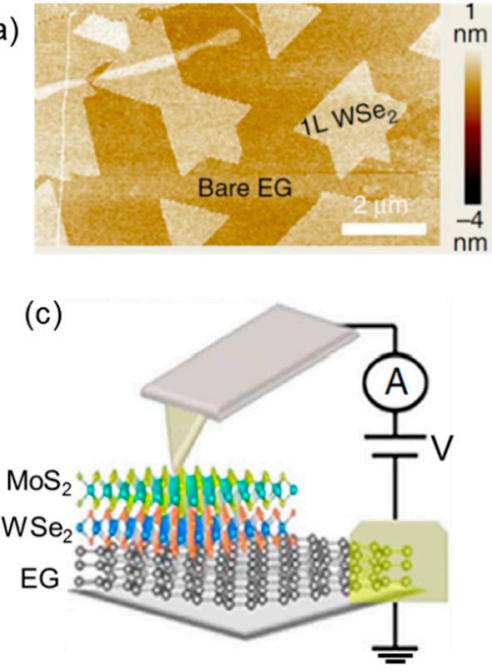

(b)
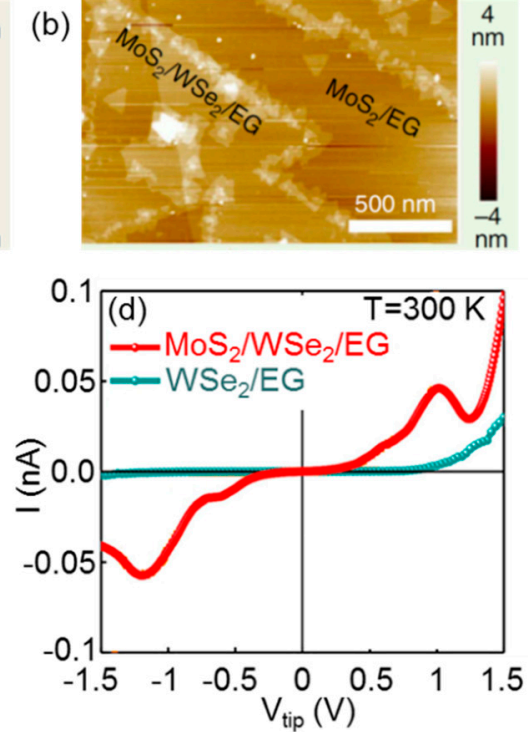

Figure 10. AFM morphology of (a) single layer $\mathrm{WSe}_{2}$ and (b) $\mathrm{MoS}_{2} / \mathrm{WSe}_{2}$ stacks deposited onto epitaxial graphene (EG) on SiC. (c) Schematic of the C-AFM setup for the I-V measurement in this layered system. (d) $\mathrm{I}-\mathrm{V}$ curves recorded at room temperature $(\mathrm{T}=300 \mathrm{~K})$ on the $\mathrm{WSe}_{2} / \mathrm{EG} \mathrm{p}-\mathrm{n}$ junction and on the $\mathrm{MoS}_{2} / \mathrm{WSe}_{2} / \mathrm{EG}$ heterostructure. Images adapted with permission from Reference [12], copyright from the Nature Publishing Group 2015.

\subsection{Vertical Heterostructures of TMDs with Bulk Semiconductors}

As shown in the previous paragraph, the Van der Waals epitaxy of heterostructures entirely made of $2 \mathrm{D}$ materials is still in its infancy, and further developments of this approach will be required to realize these systems on a large area. On the other hand, the integration of 2D materials with conventional bulk semiconductors can represent an easier root toward the exploitation of these low dimensional materials in (opto)electronics. In fact, this approach allows adding new functionalities to the existing semiconductor devices, and opening the way to the demonstration of new device concepts [11,32,93]. In the last years, the integration of graphene and semiconducting TMDs with silicon and other semiconductors, such as $\mathrm{GaN}$ and related materials [94], has been explored by several research groups. Different approaches have been explored, from the transfer of 2D materials [95-98] to the direct growth on the semiconductor substrate $[19,99]$. In particular, the epitaxial growth of $\mathrm{MoS}_{2}$ on the basal plane of GaN is especially favored by the low in-plane lattice mismatch $(<1 \%)$ between 
the two hexagonal crystals. Furthermore, the small difference in the thermal expansion coefficients between the two materials is expected to result in a reduced strain during cooling down from the growth temperature of $\mathrm{MoS}_{2}$ to room temperature [100,101].

Recently, Ruzmetov et al. [19] reported the CVD growth of epitaxially oriented $\mathrm{MoS}_{2}$ islands on the GaN basal plane. Figure 11a reports a scanning electron microscopy (SEM) image of the as-grown $\mathrm{MoS}_{2}$ on the GaN surface, consisting of triangular domains of monolayer $\mathrm{MoS}_{2}$ with a typical size of $\sim 1 \mu \mathrm{m}$. The sides of these triangles were perfectly aligned with the m-plane (1-100) of the wurzite $\mathrm{GaN}$ substrate, indicating the in-plane epitaxial alignment of the $\mathrm{GaN}$ and $\mathrm{MoS}_{2}$ lattices. Thanks to this rotational order, no evidence of grain boundaries was observed in a larger size of monolayer $\mathrm{MoS}_{2}$ islands formed by the coalescence of these small domains. This is a major advantage of $\mathrm{MoS}_{2}$ grown on GaN with respect to the more commonly used $\mathrm{CVD} \mathrm{MoS}$ on amorphous $\mathrm{SiO}_{2}$, which is a polycrystalline material with a large density of grain boundaries. In fact, as discussed in Section 3 , grain boundaries are one of the main sources of electron mobility degradation in $\mathrm{MoS}_{2}$ [81,85].

(a)

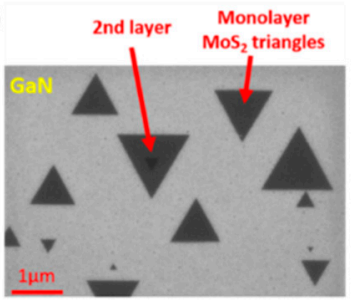

(d)

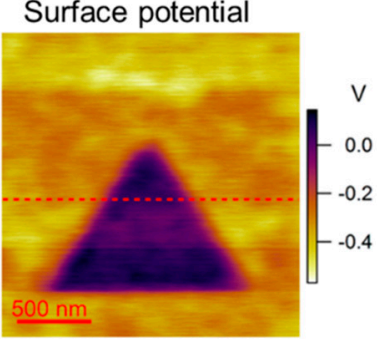

(b)

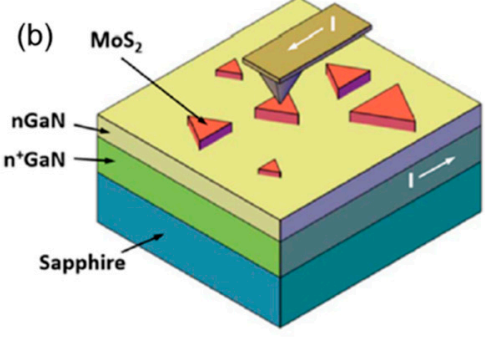

(e)

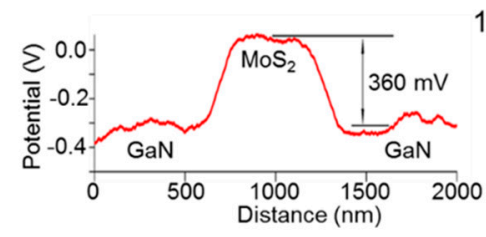

(c)

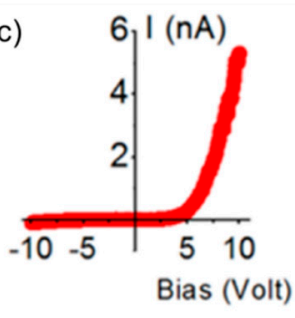

(f)

$1 \mathrm{LMOS}$

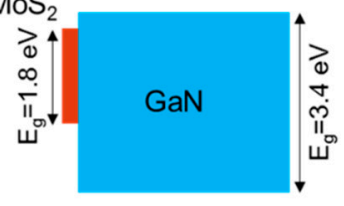

Figure 11. (a) SEM image of triangular domains of epitaxial monolayer $\mathrm{MoS}_{2}$ grown by CVD on GaN. (b) Schematic of the C-AFM setup for local I-V analyses on the $\mathrm{MoS}_{2} / \mathrm{GaN}$ junction. (c) Current-voltage characteristic on an individual $\mathrm{MoS}_{2}$ domain. (d) Surface potential map measured by KPFM on a monolayer (1L) $\mathrm{MoS}_{2}$ domain on $\mathrm{GaN}$ and (e) potential line-scan along the dashed line in the map, showing a 360-mV surface potential difference between $1 \mathrm{~L} \mathrm{MoS}_{2}$ and GaN. (f) Energy band alignment at the $1 \mathrm{~L} \mathrm{MoS}_{2} / \mathrm{GaN}$ interface, as deduced from the surface potential map. Images adapted with permission from Reference [19], copyright from the American Chemical Society 2016.

C-AFM has been applied in combination with KPFM to investigate the electrical properties of the heterojunction between $\mathrm{CVD} \mathrm{MoS}_{2}$ and n-GaN, specifically the vertical current flow across the heterointerface and the surface potential [19]. Figure 11b schematically illustrates the C-AFM setup for local current measurements, and Figure 11c reports a current vs. tip bias characteristic on an

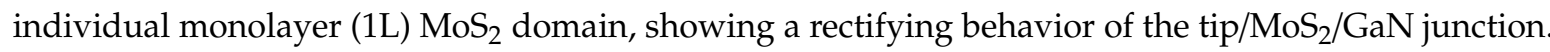
Figure $11 \mathrm{~d}$ shows a KPFM surface potential map on a GaN region partially covered by the $1 \mathrm{~L} \mathrm{MoS}_{2}$ domain. A line-scan of the surface potential along the red dashed line in the map is reported in Figure 11e, from which a surface potential difference of $\approx 360 \mathrm{meV}$ between $\mathrm{MoS}_{2}$ and $\mathrm{n}-\mathrm{GaN}$ can be evaluated [19]. Finally, Figure $11 \mathrm{f}$ shows an illustrative energy band diagram showing the type I energy band alignment between $1 \mathrm{~L} \mathrm{MoS}_{2}$ and $\mathrm{GaN}$, as deduced from the surface potential map. This peculiar energy band alignment of the $\mathrm{MoS}_{2} / \mathrm{GaN}$ heterojunction has been recently exploited in various device demonstrators, including Esaki diodes [102] and high responsivity deep-UV photodetectors [103]. 


\section{Conclusions and Perspectives}

The electronic transport properties of semiconducting TMD layers are strongly dependent on point and extended defects present in their crystalline structure. In this context, the C-AFM technique proved an essential tool to investigate the current transport in these materials at nanoscale. In this paper, recent C-AFM studies on TMDs have been reviewed, discussing the implications of local transport phenomena in the overall behavior of TMD-based devices. The current mapping and I-V spectroscopy by C-AFM allowed clarifying the mechanisms responsible for the Fermi level pinning commonly observed at the metal/TMDs interface. In particular, subsurface defects in the transition metal layer (with a density of $10^{10}-10^{11} \mathrm{~cm}^{-2}$ ) have been identified as the main origin of the pinning, although the metal/TMD interaction was also shown to partially contribute to this effect. The impact of extended defects, such as grain boundaries, on the conductivity of monolayer $\mathrm{MoS}_{2}$ grown by CVD was also directly imaged by C-AFM. Methods for nanoscale tailoring of the Schottky barrier in $\mathrm{MoS}_{2}$, such as oxygen plasma functionalization, have been illustrated, and the changes in the barrier height spatial distribution with the plasma exposure time was imaged at the nanoscale. The application of this approach for the realization of ambipolar $\mathrm{MoS}_{2}$ transistors has been also discussed. Finally, C-AFM studies of the current transport in lateral and vertical heterostructures based on TMDs have been reported. As an example, a high-resolution current mapping of $\mathrm{Mo}_{x} \mathrm{~W}_{1-\mathrm{x}} \mathrm{Se}_{2}$ alloys showed the formation of 1D lateral heterojunctions between the nanoscale Mo- and W-rich regions formed due to phase segregation. Finally, the C-AFM-based current-voltage spectroscopy provided insight on the current injection mechanisms in vertical heterojunctions formed by van der Waals epitaxy of different TMDs (such as the $\mathrm{MoS}_{2} / \mathrm{WSe}_{2}$ junction) and by the integration of TMDs with bulk semiconductors (such as the $\mathrm{MoS}_{2} / \mathrm{GaN}$ junction). Potential applications of these heterojunctions for novel electronic and optoelectronic devices have been also discussed.

Further progress in the development of $2 \mathrm{D} / 2 \mathrm{D}$ or $2 \mathrm{D} / 3 \mathrm{D}$ van der Waals heterostructures is expected in the forthcoming years. As a matter of fact, realizing the full potential of these material systems will require understanding and controlling disorder, which can obscure intrinsic properties and hinder device performances [104]. Furthermore, atomic defects or disorder can also be harnessed to provide useful electronic, optical, chemical, and magnetic functions. In this context, the C-AFM technique, in combination with other electrical and optical scanning probe methods, will represent a valuable tool to elucidate the correlation between nanoscale and macroscopic properties of the heterostructures, and will provide a guidance to tailor materials properties.

Author Contributions: Writing-original draft preparation, F.G.; writing—review and editing, F.G., E.S., G.G., and F.R.; supervision, F.G.; funding acquisition, F.G. and F.R. All authors have read and agreed to the published version of the manuscript.

Funding: This work has been funded, in part, by the FlagERA-JTC 2015 project "GraNite" and by the FlagERA-JTC 2019 project "ETMOS".

Acknowledgments: The authors want to acknowledge P. Fiorenza, R. Lo Nigro, S. Di Franco, I. Deretzis, G. Nicotra, C. Spinella, A. La Magna (CNR-IMM Catania, Italy), S. Agnello (University of Palermo, Italy), M. Bosi (CNR-IMEM, Parma, Italy), F. Fabbri (CNR-Nano, Pisa, Italy), B. Pecz (Hungarian Academy of Sciences, Budapest), A. Michon, and Y. Cordier (CNRS-CRHEA, France) for useful discussions and the participation in some of the experiments reported in this review paper.

Conflicts of Interest: The authors declare no conflict of interest.

\section{References}

1. Wang, Q.H.; Kalantar-Zadeh, K.; Kis, A.; Coleman, J.N.; Strano, M.S. Electronics and Optoelectronics of Two-Dimensional Transition Metal Dichalcogenides. Nat. Nanotechnol. 2012, 7, 699. [CrossRef]

2. Duan, X.; Wang, C.; Pan, A.; Yu, R.; Duan, X. Two-Dimensional Transition Metal Dichalcogenides as Atomically Thin Semiconductors: Opportunities and Challenges. Chem. Soc. Rev. 2015, 44, 8859-8876. [CrossRef] 
3. Splendiani, A.; Sun, L.; Zhang, Y.; Li, T.; Kim, J.; Chim, C.-Y.; Galli, G.; Wang, F. Emerging Photoluminescence in Monolayer $\mathrm{MoS}_{2}$. Nano Lett. 2010, 10, 1271-1275. [CrossRef] [PubMed]

4. Radisavljevic, B.; Radenovic, A.; Brivio, J.; Giacometti, V.; Kis, A. Single-Layer MoS $_{2}$ Transistors. Nat. Nanotechnol. 2011, 6, 147-150. [CrossRef]

5. Kim, S.; Konar, A.; Hwang, W.S.; Lee, J.H.; Lee, J.; Yang, J.; Jung, C.; Kim, H.; Yoo, J.B.; Choi, J.Y.; et al. High-Mobility and Low-Power Thin-Film Transistors Based on Multilayer $\mathrm{MoS}_{2}$ Crystals. Nat. Commun. 2012, 3, 1011. [CrossRef] [PubMed]

6. Li, M.-Y.; Su, S.-K.; Wong, H.-S.P.; Li, L.-J. How 2D semiconductors could extend Moore's law. Nature 2019, 567, 169-170. [CrossRef] [PubMed]

7. Desai, S.B.; Madhvapathy, S.R.; Sachid, A.B.; Llinas, J.P.; Wang, Q.; Ahn, G.H.; Pitner, G.; Kim, M.J.; Bokor, J.; $\mathrm{Hu}, \mathrm{C}$; et al. $\mathrm{MoS}_{2}$ Transistors with 1-Nanometer Gate Lengths. Science 2016, 354, 99-102. [CrossRef] [PubMed]

8. Nourbakhsh, A.; Zubair, A.; Sajjad, R.N.; Tavakkoli, K.G.A.; Chen, W.; Fang, S.; Ling, X.; Kong, J.; Dresselhaus, M.S.; Kaxiras, E.; et al. $\mathrm{MoS}_{2}$ Field-Effect Transistor with Sub-10 nm Channel Length. Nano Lett. 2016, 16, 7798-7806. [CrossRef]

9. Alam, K.; Lake, R.K. Monolayer $\mathrm{MoS}_{2}$ Transistors Beyond the Technology Road Map. IEEE Trans. Electron Devices 2012, 59, 3250-3254. [CrossRef]

10. Geim, A.K.; Grigorieva, I.V. Van der Waals heterostructure. Nature 2013, 499, 419-425. [CrossRef]

11. Giannazzo, F.; Greco, G.; Roccaforte, F.; Sonde, S.S. Vertical transistors based on 2D materials: Status and prospects. Crystals 2018, 8, 70. [CrossRef]

12. Lin, Y.-C.; Ghosh, R.K.; Addou, R.; Lu, N.; Eichfeld, S.M.; Zhu, H.; Li, M.-Y.; Peng, M.X.; Kim, J.; Li, L.-J.; et al. Atomically thin resonant tunnel diodes built from synthetic van der Waals heterostructures. Nat. Commun. 2015, 6, 7311.

13. Gong, Y.; Lin, J.; Wang, X.; Shi, G.; Lei, S.; Lin, Z.; Zou, X.; Ye, G.; Vajtai, R.; Yakobson, B.I.; et al. Vertical and in-plane heterostructures from $\mathrm{WS}_{2} / \mathrm{MoS}_{2}$ monolayers. Nat. Mater. 2014, 13, 1135-1142.

14. Giannazzo, F. Engineering 2D heterojunctions with dielectrics. Nat. Electron. 2019, 2, 54-55.

15. Lee, Y.-H.; Zhang, X.Q.; Zhang, W.; Chang, M.T.; Lin, C.T.; Chang, K.D.; Yu, Y.C.; Wang, J.T.; Chang, C.S.; Li, L.J.; et al. Synthesis of large-area $\mathrm{MoS}_{2}$ atomic layers with chemical vapor deposition. Adv. Mater. 2012, 24, 2320-2325. [CrossRef]

16. Yu, Y.; Li, C.; Liu, Y.; Su, L.; Zhang, Y.; Cao, L. Controlled scalable synthesis of uniform, high-quality monolayer and few-layer $\mathrm{MoS}_{2}$ Films. Sci. Rep. 2013, 3, 1866. [CrossRef]

17. Zhan, Y.; Liu, Z.; Najmaei, S.; Ajayan, P.; Lou, J. Large-area vapor-phase growth and characterization of $\mathrm{MoS}_{2}$ atomic layers on a $\mathrm{SiO}_{2}$ substrate. Small 2014, 8, 966-971. [CrossRef]

18. Dumcenco, D.; Ovchinnikov, D.; Marinov, K.; Lazic, P.; Gibertini, M.; Marzari, N.; Lopez Sanchez, O.; Kung, Y.-C.; Krasnozhon, D.; Chen, M.-W.; et al. Large-Area Epitaxial Monolayer $\mathrm{MoS}_{2}$. ACS Nano 2015, 9, 4611-4620. [CrossRef]

19. Ruzmetov, D.; Zhang, K.; Stan, G.; Kalanyan, B.; Bhimanapati, G.R.; Eichfeld, S.M.; Burke, R.A.; Shah, P.B.; O'Regan, T.P.; Crowne, F.J.; et al. Vertical 2D/3D Semiconductor Heterostructures Based on Epitaxial Molybdenum Disulfide and Gallium Nitride. ACS Nano 2016, 10, 3580-3588. [CrossRef]

20. Kang, K.; Xie, S.; Huang, L.; Han, Y.; Huang, P.Y.; Mak, K.F.; Kim, C.-J.; Muller, D.; Park, J. High-mobility three-atom-thick semiconducting films with wafer-scale homogeneity. Nature 2015, 520, 656-660. [CrossRef]

21. Yang, P.; Zou, X.; Zhang, Z.; Hong, M.; Shi, J.; Chen, S.; Shu, J.; Zhao, L.; Jiang, S.; Zhou, X.; et al. Batch production of 6-inch uniform monolayer molybdenum disulfide catalyzed by sodium in glass. Nat. Commun. 2018, 9, 979. [CrossRef] [PubMed]

22. Tan, L.K.; Liu, B.; Teng, J.H.; Guo, S.; Low, H.Y.; Loh, K.P. Atomic layer deposition of a $\mathrm{MoS}_{2}$ film. Nanoscale 2014, 6, 10584. [CrossRef] [PubMed]

23. Barton, A.T.; Yue, R.; Anwar, S.; Zhu, H.; Peng, X.; McDonnell, S.; Lu, N.; Addou, R.; Colombo, L.; Kim, M.J.; et al. Transition metal dichalcogenide and hexagonal boron nitride heterostructures grown by molecular beam epitaxy. Microelectron. Eng. 2015, 147, 306-309. [CrossRef]

24. Serna, M.I.; Yoo, S.H.; Moreno, S.; Xi, Y.; Oviedo, J.P.; Choi, H.; Alshareef, H.N.; Kim, M.J.; Minary-Jolandan, M.; Quevedo-Lopez, M.A. Large-Area Deposition of $\mathrm{MoS}_{2}$ by Pulsed Laser Deposition with In Situ Thickness Control. ACS Nano 2016, 10, 6054-6061. [CrossRef] [PubMed] 
25. Radisavljevic, B.; Whitwick, M.B.; Kis, A. Integrated circuits and logic operations based on single-layer $\mathrm{MoS}_{2}$. Nano Lett. 2011, 5, 9934-9938. [CrossRef] [PubMed]

26. Fang, H.; Chuang, S.; Chang, T.C.; Takei, K.; Takahashi, T.; Javey, A. High-Performance Single Layered WSe 2 p-FETs with Chemically Doped Contacts. Nano Lett. 2012, 12, 3788-3792. [CrossRef] [PubMed]

27. Roy, T.; Tosun, M.; Kang, J.K.; Sachid, A.B.; Desai, S.B.; Hettick, M.; Hu, C.C.; Javey, A. Field-Effect Transistors Built from All Two-Dimensional Material Components. ACS Nano 2014, 8, 6259-6264. [CrossRef]

28. Yu, L.; Zubair, A.; Santos, E.J.G.; Zhang, X.; Lin, Y.; Zhang, Y.; Palacios, T. High performance WSe ${ }_{2}$ complementary metal oxide semiconductor technology and integrated circuits. Nano Lett. 2015, 15, 4928-4934. [CrossRef]

29. Giannazzo, F.; Fisichella, G.; Greco, G.; Di Franco, S.; Deretzis, I.; La Magna, A.; Bongiorno, C.; Nicotra, G.; Spinella, C.; Scopelliti, M.; et al. Ambipolar $\mathrm{MoS}_{2}$ Transistors by Nanoscale Tailoring of Schottky Barrier Using Oxygen Plasma Functionalization. ACS Appl. Mater. Interfaces 2017, 9, 23164-23174. [CrossRef]

30. Cui, X.; Shih, E.-M.; Jauregui, L.A.; Chae, S.H.; Kim, Y.D.; Li, B.; Seo, D.; Pistunova, K.; Yin, J.; Park, J.-H.; et al. Low-Temperature Ohmic Contact to Monolayer $\mathrm{MoS}_{2}$ by van der Waals Bonded Co/h-BN Electrodes. Nano Lett. 2017, 17, 4781-4786. [CrossRef]

31. Wachter, S.; Polyushkin, D.K.; Bethge, O.; Mueller, T. A microprocessor based on a two-dimensional semiconductor. Nat. Commun. 2017, 8, 14948. [CrossRef] [PubMed]

32. Zubair, A.; Nourbakhsh, A.; Hong, J.-Y.; Qi, M.; Song, Y.; Jena, D.; Kong, J.; Dresselhaus, M.; Palacios, T. Hot Electron Transistor with van der Waals Base-Collector Heterojunction and High- Performance GaN Emitter. Nano Lett. 2017, 17, 3089-3096. [CrossRef]

33. Wang, Y.; Kim, J.C.; Wu, R.J.; Martinez, J.; Song, X.; Yang, J.; Zhao, F.; Mkhoyan, A.; Jeong, H.Y.; Chhowalla, M. Van der Waals contacts between three-dimensional metals and two-dimensional semiconductors. Nature 2019, 568, 70-74. [CrossRef]

34. Giannazzo, F.; Raineri, V.; Rimini, E. Transport properties of graphene with nanoscale lateral resolution. In Scanning Probe Microscopy in Nanoscience and Nanotechnology; Bhushan, B., Ed.; Springer: Berlin/Heidelberg, Germany, 2011; Volume 2.

35. Giannazzo, F.; Sonde, S.; Raineri, V.; Rimini, E. Irradiation damage in graphene on $\mathrm{SiO}_{2}$ probed by local mobility measurements. Appl. Phys. Lett. 2009, 95, 263109.

36. Giannazzo, F.; Sonde, S.; Raineri, V.; Rimini, E. Screening Length and Quantum Capacitance in Graphene by Scanning Probe Microscopy. Nano Lett. 2009, 9, 23. [CrossRef] [PubMed]

37. Sonde, S.; Giannazzo, F.; Vecchio, C.; Yakimova, R.; Rimini, E.; Raineri, V. Role of graphene/substrate interface on the local transport properties of the two-dimensional electron gas. Appl. Phys. Lett. 2010, 97, 132101. [CrossRef]

38. Giannazzo, F.; Sonde, S.; Lo Nigro, R.; Rimini, E.; Raineri, V. Mapping the Density of Scattering Centers Limiting the Electron Mean Free Path in Graphene. Nano Lett. 2011, 11, 4612-4618. [CrossRef]

39. Wu, D.; Li, X.; Luan, L.; Wu, X.; Li, W.; Yogeesh, M.N.; Ghosh, R.; Chu, Z.; Akinwande, D.; Niu, Q.; et al. Uncovering Edge States and Electrical Inhomogeneity in $\mathrm{MoS}_{2}$ Field-Effect Transistors. Proc. Natl. Acad. Sci. USA 2016, 113, 8583-8588. [CrossRef]

40. Giannazzo, F.; Fisichella, G.; Greco, G.; Fiorenza, P.; Roccaforte, F. Conductive Atomic Force Microscopy of Two-Dimensional Electron Systems: From AlGaN/GaN Heterostructures to Graphene and $\mathrm{MoS}_{2}$. In Conductive Atomic Force Microscopy: Applications in Nanomaterials; Lanza, M., Ed.; WILEY-VCH Verlag: Weinheim, Germany, 2017; Chapter 7; pp. 163-186.

41. Lanza, M. Conductive Atomic Force Microscopy: Applications in Nanomaterials; Wiley-VCH Verlag: Weinheim, Germany, 2017.

42. Giannazzo, F.; Greco, G.; Roccaforte, F.; Mahata, C.; Lanza, M. Conductive AFM of 2D Materials and Heterostructures for Nanoelectronics. In Electrical Atomic Force Microscopy for Nanoelectronics; Celano., U., Ed.; Springer: Berlin, Germany, 2019; Chapter 10; pp. 303-350.

43. Hui, F.; Lanza, M. Scanning probe microscopy for advanced nanoelectronics. Nat. Electron. 2019, 2, 221. [CrossRef]

44. Giannazzo, F.; Fisichella, G.; Piazza, A.; Agnello, S.; Roccaforte, F. Nanoscale Inhomogeneity of the Schottky Barrier and Resistivity in $\mathrm{MoS}_{2}$ Multilayers. Phys. Rev. B 2015, 92, 081307(R). [CrossRef] 
45. Bampoulis, P.; van Bremen, R.; Yao, Q.; Poelsema, B.; Zandvliet, H.J.W.; Sotthewes, K. Defect Dominated Charge Transport and Fermi Level Pinning in $\mathrm{MoS}_{2} /$ Metal Contacts. ACS Appl. Mater. Interfaces 2017, 9 , 19278-19286. [CrossRef] [PubMed]

46. Giannazzo, F.; Deretzis, I.; La Magna, A.; Roccaforte, F.; Yakimova, R. Electronic transport at monolayer-bilayer junctions in epitaxial graphene on SiC. Phys. Rev. B 2012, 86, 235422. [CrossRef]

47. Nicotra, G.; Ramasse, Q.M.; Deretzis, I.; La Magna, A.; Spinella, C.; Giannazzo, F. Delaminated Graphene at Silicon Carbide Facets: Atomic Scale Imaging and Spectroscopy. ACS Nano 2013, 7, 3045-3052. [CrossRef] [PubMed]

48. Vasic, B.; Zurutuza, A.; Gajic, R. Spatial variation of wear and electrical properties across wrinkles in chemical vapour deposition graphene. Carbon 2016, 102, 304-310. [CrossRef]

49. Fisichella, G.; Schilirò, E.; Di Franco, S.; Fiorenza, P.; Lo Nigro, R.; Roccaforte, F.; Ravesi, S.; Giannazzo, F. Interface Electrical Properties of $\mathrm{Al}_{2} \mathrm{O}_{3}$ Thin Films on Graphene Obtained by Atomic Layer Deposition with an in Situ Seedlike Layer. ACS Appl. Mater. Interfaces 2017, 9, 7761-7771. [CrossRef]

50. Schilirò, E.; Lo Nigro, R.; Roccaforte, F.; Deretzis, J.; La Magna, A.; Armano, A.; Agnello, S.; Pecz, B.; Ivanov, I.G.; Giannazzo, F. Seed-Layer-Free Atomic Layer Deposition of Highly Uniform $\mathrm{Al}_{2} \mathrm{O}_{3}$ Thin Films onto Monolayer Epitaxial Graphene on Silicon Carbide. Adv. Mater. Interfaces 2019, 6, 1900097. [CrossRef]

51. Britnell, L.; Gorbachev, R.V.; Jalil, R.; Belle, B.D.; Schedin, F.; Katsnelson, M.I.; Eaves, L.; Morozov, S.V.; Mayorov, A.S.; Peres, N.M.R.; et al. Electron Tunneling through Ultrathin Boron Nitride Crystalline Barriers. Nano Lett. 2012, 12, 1707-1710. [CrossRef]

52. Lee, G.H.; Yu, Y.J.; Lee, C.G.; Dean, C.; Shepard, K.L.; Kim, P.; Hone, J. Electron tunneling through atomically flat and ultrathin hexagonal boron nitride. Appl. Phys. Lett. 2011, 99, 243114. [CrossRef]

53. Hui, F.; Fang, W.; Leong, W.S.; Kpulun, T.; Wang, H.; Yang, H.Y.; Villena, M.A.; Harris, G.; Kong, J.; Lanza, M. Electrical Homogeneity of Large-Area Chemical Vapor Deposited Multilayer Hexagonal Boron Nitride Sheets. ACS Appl. Mater. Interfaces. 2017, 9, 39895-39900. [CrossRef]

54. Sonde, S.; Giannazzo, F.; Raineri, V.; Yakimova, R.; Huntzinger, J.-R.; Tiberj, A.; Camassel, J. Electrical properties of the graphene/4H-SiC (0001) interface probed by scanning current spectroscopy. Phys. Rev. B 2009, 80, 241406. [CrossRef]

55. Bouhafs, C.; Zakharov, A.A.; Ivanov, I.G.; Giannazzo, F.; Eriksson, J.; Stanishev, V.; Kühne, P.; Iakimov, T.; Hofmann, T.; Schubert, M.; et al. Multi-scale investigation of interface properties, stacking order and decoupling of few layer graphene on C-face $4 \mathrm{H}-\mathrm{SiC}$. Carbon 2017, 116, 722-732. [CrossRef]

56. Fisichella, G.; Greco, G.; Roccaforte, F.; Giannazzo, F. Current transport in graphene/AlGaN/GaN vertical heterostructures probed at nanoscale. Nanoscale 2014, 6, 8671-8680. [CrossRef] [PubMed]

57. Lince, J.R.; Carré, D.J.; Fleischauer, P.D. Schottky-Barrier Formation on a Covalent Semiconductor without Fermi-Level Pinning: The Metal-MoS 2 (0001) Interface. Phys. Rev. B 1987, 36, 1647-1656. [CrossRef] [PubMed]

58. Das, S.; Chen, H.-Y.; Penumatcha, A.V.; Appenzeller, J. High Performance Multi-layer $\mathrm{MoS}_{2}$ Transistors with Scandium Contacts. Nano Lett. 2012, 13, 100. [CrossRef] [PubMed]

59. Guo, Y.; Liu, D.; Robertson, J. 3D Behavior of Schottky Barriers of 2D Transition-Metal Dichalcogenides. ACS Appl. Mater. Interfaces 2015, 7, 25709. [CrossRef] [PubMed]

60. Kim, C.; Moon, I.; Lee, D.; Choi, M.S.; Ahmed, F.; Nam, S.; Cho, Y.; Shin, H.-J.; Park, S.; Yoo, W.J. Fermi Pinning at Electrical Metal Contacts of Monolayer Molybdenum Dichalcogenides. ACS Nano 2017, 11, 1588-1596. [CrossRef]

61. Gong, C.; Colombo, L.; Wallace, R.M.; Cho, K. The Unusual Mechanism of Partial Fermi Level Pinning at Metal-MoS 2 Interfaces. Nano Lett. 2014, 14, 1714. [CrossRef]

62. Giannazzo, F.; Fisichella, G.; Piazza, A.; Di Franco, S.; Greco, G.; Agnello, S.; Roccaforte, F. Impact of Contact Resistance on the Electrical Properties of $\mathrm{MoS}_{2}$ Transistors at Practical Operating Temperatures. Beilstein J. Nanotechnol. 2017, 8, 254-263. [CrossRef]

63. Das, S.; Prakash, A.; Salazar, R.; Appenzeller, J. Toward Low-Power Electronics: Tunneling Phenomena in Transition Metal Dichalcogenides. ACS Nano 2014, 8, 1681-1689. [CrossRef]

64. Giannazzo, F.; Fisichella, G.; Piazza, A.; Di Franco, S.; Greco, G.; Agnello, S.; Roccaforte, F. Effect of Temperature-Bias Annealing on the Hysteresis and Subthreshold Behavior of Multilayer $\mathrm{MoS}_{2}$ Transistors. Phys. Status Solidi RRL 2016, 10, 797-801. [CrossRef] 
65. Lin, Y.-F.; Xu, Y.; Wang, S.-T.; Li, S.-L.; Yamamoto, M.; Aparecido-Ferreira, A.; Li, W.; Sun, H.; Nakaharai, S.; Jian, W.-B.; et al. Ambipolar MoTe 2 Transistors and Their Applications in Logic Circuits. Adv. Mater. 2014, 26, 3263-3269. [CrossRef] [PubMed]

66. Heine, V. Theory of Surface States. Phys. Rev. 1965, 138, A1689. [CrossRef]

67. Hasegawa, H.; Ohno, H. Unified Disorder Induced Gap State Model for Insulator-Semiconductor and Metal-Semiconductor Interfaces. J. Vac. Sci. Technol. B Microelectron. Process. Phenom. 1986, 4, 1130-1138. [CrossRef]

68. McDonnell, S.; Addou, R.; Buie, C.; Wallace, R.M.; Hinkle, C.L. Defect-Dominated Doping and Contact Resistance in $\mathrm{MoS}_{2}$. ACS Nano 2014, 8, 2880-2888. [CrossRef] [PubMed]

69. Bampoulis, P.; Sotthewes, K.; Siekman, M.H.; Zandvliet, H.J.W. Local Conduction in $\mathrm{Mo}_{x} \mathrm{~W}_{1-\mathrm{x}} \mathrm{Se}_{2}$ : The Role of Stacking Faults, Defects, and Alloying. ACS Appl. Mater. Interfaces 2018, 10, 13218-13225. [CrossRef]

70. Smyth, C.M.; Addou, R.; McDonnell, S.; Hinkle, C.L.; Wallace, R.M. Contact Metal-MoS 2 Interfacial Reactions and Potential Implications on $\mathrm{MoS}_{2}$-Based Device Performance. J. Phys. Chem. C 2016, 120, 14719-14729. [CrossRef]

71. Kim, C.; Lee, K.Y.; Moon, I.; Issarapanacheewin, S.; Yoo, W.J. Metallic contact induced van der Waals gap in a $\mathrm{MoS}_{2}$ FET. Nanoscale 2019, 11, 18246. [CrossRef]

72. Cheung, S.K.; Cheung, N.W. Extraction of Schottky diode parameters from forward current-voltage characteristics. Appl. Phys. Lett. 1986, 49, 85.

73. Card, H.; Rhoderick, E.H. Studies of tunnel MOS diodes II. Thermal equilibrium considerations. J. Phys. D 1971, 4, 1589.

74. Aguilera, L.; Polspoel, W.; Volodin, A.; Van Haesendonck, C.; Porti, M.; Vandervorst, W.; Nafria, M.; Aymerich, $X$. Influence of vacuum environment on conductive atomic force microscopy measurements of advanced metal-oxide-semiconductor gate dielectrics. J. Vac. Sci. Technol. B 2008, 26, 1445. [CrossRef]

75. Sotthewes, K.; van Bremen, R.; Dollekamp, E.; Boulogne, T.; Nowakowski, K.; Kas, D.; Zandvliet Harold, J.W.; Bampoulis, P. Universal Fermi-Level Pinning in Transition-Metal Dichalcogenides. J. Phys. Chem. C 2019, 123, 5411. [CrossRef] [PubMed]

76. Allain, A.; Kang, J.; Banerjee, K.; Kis, A. Electrical Contacts to Two-Dimensional Semiconductors. Nat. Mater. 2015, 14, 1195. [CrossRef] [PubMed]

77. Van der Zande, A.M.; Huang, P.Y.; Chenet, D.A.; Berkelbach, T.C.; You, Y.; Lee, G.-H.; Heinz, T.F.; Reichman, D.R.; Muller, D.A.; Hone, J.C. Grains and grain boundaries in highly crystalline monolayer molybdenum disulphide. Nat. Mater. 2013, 12, 554-561.

78. Karvonen, L.; Säynätjoki, A.; Huttunen, M.J.; Autere, A.; Amirsolaimani, B.; Li, S.; Norwood, R.A.; Peyghambarian, N.; Lipsanen, H.; Eda, G.; et al. Rapid visualization of grain boundaries in monolayer $\mathrm{MoS}_{2}$ by multiphoton microscopy. Nat. Commun. 2017, 8, 15714. [CrossRef] [PubMed]

79. Majee, A.K.; Foss, C.J.; Aksamija, Z. Impact of Mismatch Angle on Electronic Transport Across Grain Boundaries and Interfaces in 2D Materials. Sci. Rep. 2017, 7, 16597. [CrossRef] [PubMed]

80. Sangwan, V.K.; Jariwala, D.; Kim, I.S.; Chen, K.-S.; Marks, T.J.; Lauhon, L.J.; Hersam, M.C. Gate-tunable memristive phenomena mediated by grain boundaries in single-layer $\mathrm{MoS}_{2}$. Nat. Nanotechnol. 2015, 10, 403-406. [CrossRef]

81. Ly, T.H.; Perello, D.J.; Zhao, J.; Deng, Q.; Kim, H.; Han, G.H.; Chae, S.H.; Jeong, H.Y.; Hee Lee, Y. Misorientation-angle-dependent electrical transport across molybdenum disulfide grain boundaries. Nat. Commun. 2016, 7, 10426. [CrossRef]

82. Lu, C.-P.; Li, G.; Mao, J.; Wang, L.-M.; Andrei, E.Y. Bandgap, Mid-Gap States, and Gating Effects in MoS 2 . Nano Lett. 2014, 14, 4628-4633. [CrossRef]

83. Qiu, H.; Xu, T.; Wang, Z.; Ren, W.; Nan, H.; Ni, Z.; Chen, Q.; Yuan, S.; Miao, F.; Song, F.; et al. Hopping transport through defect-induced localized states in molybdenum disulphide. Nat. Commun. 2013, 4, 2642. [CrossRef]

84. Ludwig, J.; Mehta, A.N.; Mascaro, M.; Celano, U.; Chiappe, D.; Bender, H.; Vandervorst, W.; Paredis, K. Effects of buried grain boundaries in multilayer $\mathrm{MoS}_{2}$. Nanotechnology 2019, 30, 285705. [CrossRef]

85. Giannazzo, F.; Bosi, M.; Fabbri, F.; Schilirò, E.; Greco, G.; Roccaforte, F. Direct Probing of Grain Boundary Resistance in Chemical Vapor Deposition-Grown Monolayer $\mathrm{MoS}_{2}$ by Conductive Atomic Force Microscopy. Phys. Status Solidi RRL 2020, 14, 1900393. [CrossRef] 
86. Zhang, C.; Chen, Y.; Huang, J.-K.; Wu, X.; Li, L.-J.; Yao, W.; Tersoff, J.; Shih, C.-K. Visualizing band offsets and edge states in bilayer-monolayer transition metal dichalcogenides lateral heterojunction. Nat. Commun. 2015, 7, 10349. [CrossRef] [PubMed]

87. Kobayashi, Y.; Mori, S.; Maniwa, Y.; Miyata, Y. Bandgap-Tunable Lateral and Vertical Heterostructures Based on Monolayer $\mathrm{Mo}_{1-\mathrm{x}} \mathrm{W}_{\mathrm{x}} \mathrm{S}_{2}$ Alloys. Nano Res. 2015, 8, 3261-3271. [CrossRef]

88. Chen, Y.; Xi, J.; Dumcenco, D.O.; Liu, Z.; Suenaga, K.; Wang, D.; Shuai, Z.; Huang, Y.-S.; Xie, L. Tunable Band Gap Photoluminescence from Atomically Thin Transition-Metal Dichalcogenide Alloys. ACS Nano 2013, 7 , 4610-4616. [CrossRef]

89. Huang, C.; Wu, S.; Sanchez, A.M.; Peters, J.J.P.; Beanland, R.; Ross, J.S.; Rivera, P.; Yao, W.; Cobden, D.H.; $\mathrm{Xu}, \mathrm{X}$. Lateral Heterojunctions within Monolayer $\mathrm{MoSe}_{2}-\mathrm{WSe}_{2}$ Semiconductors. Nat. Mater. 2014, 13, 1096-1101. [CrossRef]

90. Feng, Q.; Zhu, Y.; Hong, J.; Zhang, M.; Duan, W.; Mao, N.; Wu, J.; Xu, H.; Dong, F.; Lin, F.; et al. Growth of Large-Area 2D MoS ${ }_{2(1-x)} \mathrm{Se}_{2 \mathrm{x}}$ Semiconductor Alloys. Adv. Mater. 2014, 26, 2648-2653. [CrossRef]

91. Susarla, S.; Kochat, V.; Kutana, A.; Hachtel, J.A.; Idrobo, J.C.; Vajtai, R.; Yakobson, B.I.; Tiwary, C.S.; Ajayan, P.M. Phase Segregation Behavior of Two-Dimensional Transition Metal Dichalcogenide Binary Alloys Induced by Dissimilar Substitution. Chem. Mater. 2017, 29, 7431-7439. [CrossRef]

92. Yoshida, S.; Kobayashi, Y.; Sakurada, R.; Mori, S.; Miyata, Y.; Mogi, H.; Koyama, T.; Takeuchi, O.; Shigekawa, H. Microscopic Basis for the Band Engineering of $\mathrm{Mo}_{1-\mathrm{x}} \mathrm{W}_{\mathrm{x}} \mathrm{S}_{2}$-Based Heterojunction. Sci. Rep. 2015, 5, 14808. [CrossRef]

93. Giannazzo, F.; Greco, G.; Schilirò, E.; Lo Nigro, R.; Deretzis, I.; La Magna, A.; Roccaforte, F.; Iucolano, F.; Ravesi, S.; Frayssinet, E.; et al. High-Performance Graphene/AlGaN/GaN Schottky Junctions for Hot Electron Transistors. ACS Appl. Electron. Mater. 2019, 1, 2342-2354. [CrossRef]

94. Giannazzo, F.; Fisichella, G.; Greco, G.; La Magna, A.; Roccaforte, F.; Pecz, B.; Yakimova, R.; Dagher, R.; Michon, A.; Cordier, Y. Graphene integration with nitride semiconductors for high power and high frequency electronics. Phys. Status Solidi A 2017, 214, 1600460. [CrossRef]

95. Kang, J.; Shin, D.; Bae, S.; Hong, B.H. Graphene transfer: Key for applications. Nanoscale 2012, 4, 5527. [CrossRef]

96. Fisichella, G.; Di Franco, S.; Roccaforte, F.; Ravesi, S.; Giannazzo, F. Microscopic mechanisms of graphene electrolytic delamination from metal substrates. Appl. Phys. Lett. 2014, 104, 233105. [CrossRef]

97. Giannazzo, F.; Fisichella, G.; Greco, G.; Schilirò, E.; Deretzis, I.; Lo Nigro, R.; La Magna, A.; Roccaforte, F.; Iucolano, F.; Lo Verso, S.; et al. Fabrication and Characterization of Graphene Heterostructures with Nitride Semiconductors for High Frequency Vertical Transistors. Phys. Status Solidi A 2018, 215, 1700653. [CrossRef]

98. Prystawko, P.; Giannazzo, F.; Krysko, M.; Smalc-Koziorowska, J.; Schilirò, E.; Greco, G.; Roccaforte, F.; Leszczynski, M. Growth and characterization of thin Al-rich AlGaN on bulk GaN as an emitter-base barrier for hot electron transistor. Mat. Sci. Semic. Proc. 2019, 93, 153-157. [CrossRef]

99. Michon, A.; Tiberj, A.; Vezian, S.; Roudon, E.; Lefebvre, D.; Portail, M.; Zielinski, M.; Chassagne, T.; Camassel, J.; Cordier, Y. Graphene growth on AlN templates on silicon using propane-hydrogen chemical vapor deposition. Appl. Phys. Lett. 2014, 104, 071912. [CrossRef]

100. Huang, L.F.; Gong, P.L.; Zeng, Z. Correlation between Structure, Phonon Spectra, Thermal Expansion, and Thermomechanics of Single-Layer MoS 2 . Phys. Rev. B 2014, 90, 045409. [CrossRef]

101. Leszczynski, M.; Suski, T.; Teisseyre, H.; Perlin, P.; Grzegory, I.; Jun, J.; Porowski, S.; Moustakas, T.D. Thermal Expansion of Gallium Nitride. J. Appl. Phys. 1994, 76, 4909-4911. [CrossRef]

102. Krishnamoorthy, S.; Lee, E.W.; Hee Lee, C.; Zhang, Y.; McCulloch, W.D.; Johnson, J.M.; Hwang, J.; Wu, Y.; Rajan, S. High current density 2D/3D MoS $/$ GaN Esaki tunnel diodes. Appl. Phys. Lett. 2016, 109, 183505. [CrossRef]

103. Zhuo, R.; Wang, Y.; Wu, D.; Lou, Z.; Shi, Z.; Xu, T.; Xu, J.; Tian, Y.; Li, X. High-performance self-powered deep ultraviolet photodetector based on $\mathrm{MoS}_{2} / \mathrm{GaN}$ p-n heterojunction. J. Mater. Chem. C 2018, 6, 299. [CrossRef]

104. Rhodes, D.; Chae, S.H.; Ribeiro-Palau, R.; Hone, J. Disorder in van der Waals heterostructures of 2D materials. Nat. Mater. 2019, 18, 541-549. [CrossRef]

(C) 2020 by the authors. Licensee MDPI, Basel, Switzerland. This article is an open access article distributed under the terms and conditions of the Creative Commons Attribution (CC BY) license (http://creativecommons.org/licenses/by/4.0/). 